\title{
ON THE CAUCHY PROBLEM ASSOCIATED WITH THE MOTION OF A BINGHAM FLUID IN THE PLANE
}

\author{
JONG UHN KIM
}

\begin{abstract}
This paper discusses an initial value problem for the variational inequality which describes the motion of a Bingham fluid in the plane. The existence of strong solution is established.
\end{abstract}

0. Introduction. The purpose of this paper is to establish the existence and uniqueness of strong solutions to the Cauchy problem associated with the motion of a Bingham fluid in $R^{2}$ :

$$
\begin{gathered}
\left\langle\frac{\partial u}{\partial t}, w-u\right\rangle+\langle(u \cdot \nabla) u, w-u\rangle+\mu a(u, w-u) \\
+\nu \int_{R^{2}} D(w) d x-\nu \int_{R^{2}} D(u) d x \geq\langle F, w-u\rangle, \\
\nabla \cdot u=0, \\
u(x, 0)=u_{0}(x),
\end{gathered}
$$

where $x=\left(x_{1}, x_{2}\right)$ is the space variable, $t$ is the time variable, $u(x, t)$ denotes the velocity vector and $F(x, t)$ is the known external force; the bracket $\langle$,$\rangle denotes$ the inner product in $L^{2}\left(R^{2}\right)^{2} ; \mu>0$ is the viscosity coefficient and $\nu>0$ is the yield limit;

$$
\begin{gathered}
\varepsilon_{i j}(u)=\frac{1}{2}\left(\frac{\partial u_{i}}{\partial x_{j}}+\frac{\partial u_{j}}{\partial x_{i}}\right), \quad i, j=1,2, \text { for } u=\left(u_{1}, u_{2}\right) ; \\
D(u)=\left(2 \sum_{i, j=1}^{2} \varepsilon_{i j}(u)^{2}\right)^{1 / 2} ; \\
a(u, v)=2 \sum_{i, j=1}^{2} \int_{R^{2}} \varepsilon_{i j}(u) \varepsilon_{i j}(v) d x
\end{gathered}
$$

for a more detailed description of notation, see the following section. Here, (0-2) is the condition of incompressibility and (0-1) is supposed to hold for almost all $t$ of a certain time interval for every $w(x)$ of a properly chosen function class, which we shall specify later on according to the definition of strong solution. Our definition of strong solution is motivated by the requirement that each term of (0-1) makes sense as an ordinary function and that the uniqueness of solution is easily proved; the precise definition is presented in the subsequent section.

Received by the editors June 14, 1985 and, in revised form, November 18, 1985.

1980 Mathematics Subject Classification (1985 Revision). Primary 35Q20, 76D99; Secondary $35 \mathrm{~A} 05$. 
A Bingham fluid is a rigid visco-plastic fluid which is described by special constituent laws such that it behaves like a rigid medium if a certain function of the stresses does not reach the yield limit, and its motion is close to that of a Newtonian fluid beyond this threshold. In fact, if $\nu=0,(0-1)$ reduces to the Navier-Stokes equations. For $\nu>0$, the flow contains rigid zones and the model is better described by the variational inequality which resolves the difficulty of interfaces separating two different zones; for the derivation of (0-1), see Duvaut and Lions [1].

The initial boundary value problem associated with (0-1) to (0-3) was discussed by Duvaut and Lions [1], where weak solutions were obtained and the regularity of weak solutions was studied in the case of space dimension two. But it is not known whether there exists so regular a solution as to allow the second derivatives of $u(x, t)$ with respect to $x$ to be square integrable over the space domain at least for almost all $t$ on a certain time interval even if the initial data and the forcing term are sufficiently smooth. Our main result Theorem 2.2 asserts that there exists such a solution which is also unique in a certain function class if the space domain is the whole space $\mathbf{R}^{2}$. When the space domain has a boundary, the major difficulty in obtaining the regularity of solutions of $(0-1)$ to $(0-3)$ lies in the fact that the projection operator associated with the Helmholtz decomposition does not commute with the Laplacian, and the standard technique of deriving energy estimates cannot be applied. On the other hand, if the space domain is the whole space, then the projection operator commutes with the Laplacian in the suitable function spaces. We are, however, faced with different trouble: we do not have a complete set of eigenfunctions associated with the Stokes operator while we can choose a complete orthonormal basis for $L^{2}\left(R^{2}\right)^{2}$. Hence, if we employ the Galerkin approximation scheme using a certain orthonormal basis, we cannot obtain enough a priori estimates for each approximating system of equations since $\Delta u$ does not belong to the same finite dimensional subspace where $u$ is chosen. Therefore, the major task for our result is to overcome these difficulties. The recipe is to partition the whole space by a countable set of identical blocks and to consider the periodic boundary condition. Then the existence of a complete set of eigenfunctions associated with the Stokes operator is ensured and, at the same time, the projection operator commutes with the Laplacian. For each period, we establish the existence of a strong solution to a regularized and space-periodic problem by the Galerkin method. By making use of the convexity of the regularized functional, we obtain sufficient a priori estimates independent of the period, i.e., the size of each block. We then pass a sequence of space-periodic solutions to a limit by expanding the block centered at the origin to the whole space. The limit function is shown to be a strong solution of the regularized problem in the whole space. Finally, we pass the regularizing parameter to the limit to arrive at a strong solution of the original problem, the uniqueness of which is also proved.

It is interesting to compare the regularity of our strong solution and that of the solution to the Navier-Stokes equations with the same initial condition. Since it is known that we can raise indefinitely the regularity of solutions to the Navier-Stokes equations by assuming more regularity on the initial data, we are naturally led to wonder if the same is true of (0-1) to (0-3). The example in $\S 4$ shows that this is not true at least in terms of the regularity in time near $t=0$. We also discuss the behavior of solutions as $\nu$ tends to zero. Using the same method as in Duvaut and 
Lions [1], it is shown that the strong solution of the above problem converges to the solution of the Navier-Stokes equations as $\nu$ tends to zero. But the convergence is weaker than that in $[\mathbf{1}]$. This is due to the fact that the $L^{1}$-norm is not dominated by the $L^{2}$-norm in an unbounded domain.

Finally we comment on the possibility of extending the present method to the case of $R^{3}$.

\section{Preliminaries.}

1.1. Notations. $\mathbf{Z}^{n}$ is the set of all $n$-tuples of integers and $\mathbf{C}^{n}$ is the set of all $n$-tuples of complex numbers. $E_{L}=\left\{\left(x_{1}, x_{2}\right):-L / 2 \leq x_{i} \leq L / 2, i=1,2\right\}$ and $C^{k}\left(E_{L}\right)$ denotes the set of all real-valued, $L$-periodic, $k$ times continuously differentiable functions in $R^{2}$. $L^{p}\left(E_{L}\right), 1 \leq p \leq \infty$, stands for the set of all realvalued, $L$-periodic functions which are locally $L^{p}$ in $R^{2}$. When $\mathbf{B}$ is a Banach space, $C([0, T] ; \mathbf{B})$ is the space of all $\mathbf{B}$-valued continuous functions on $[0, T]$, and $L^{p}(0, T ; \mathbf{B})$ is the space of all $\mathbf{B}$-valued, strongly measurable, $L^{p}$ functions on $[0, T]$. For $\alpha=\left(\alpha_{1}, \ldots, \alpha_{n}\right), \beta=\left(\beta_{1}, \ldots, \beta_{n}\right) \in \mathbf{C}^{n}$, we use the notation $\alpha \cdot \beta=\sum_{i=1}^{n} \alpha_{i} \beta_{i}$ and $|\alpha|=\left(\sum_{i=1}^{n}\left|\alpha_{i}\right|^{2}\right)^{1 / 2}$. For a real-valued function $g(x)$ in $R^{2}$, we write

and

$$
\nabla g=\left(\frac{\partial g}{\partial x_{1}}, \frac{\partial g}{\partial x_{2}}\right)
$$

$$
|\nabla g|=\left(\sum_{i=1}^{2}\left(\frac{\partial g}{\partial x_{i}}\right)^{2}\right)^{1 / 2}
$$

For $f=\left(f_{1}, f_{2}\right)$ an $R^{2}$-valued function in $R^{2}$, we let

$$
\begin{gathered}
\varepsilon_{i j}(f)=\frac{1}{2}\left(\frac{\partial f_{i}}{\partial x_{j}}+\frac{\partial f_{j}}{\partial x_{i}}\right), \quad i, j=1,2 \\
D(f)=\left(2 \sum_{i, j=1}^{2} \varepsilon_{i j}(f)^{2}\right)^{1 / 2}, \quad \nabla \cdot f=\sum_{i=1}^{2} \frac{\partial f_{i}}{\partial x_{i}} \\
|\nabla f|=\left(\sum_{i, j=1}^{2}\left(\frac{\partial f_{i}}{\partial x_{j}}\right)^{2}\right)^{1 / 2}, \quad\|f\|_{L^{p}(G)}=\||f|\|_{L^{p}(G)}
\end{gathered}
$$

and

$$
\|\nabla f\|_{L^{p}(G)}=\||\nabla f|\|_{L^{p}(G)}
$$

where $G$ is a measurable subset of $R^{2}$. When $\Omega$ is an open subset of $R^{n}, D^{\prime}(\Omega)$ denotes the space of distributions in $\Omega$ and $W^{m, p}(\Omega), m=$ nonnegative integer, consists of all real-valued $L^{p}(\Omega)$ functions which possess generalized derivatives up to order $m$ in $L^{p}(\Omega) . W^{-m, q}(\Omega), m=$ nonnegative integer, $1<q \leq \infty$, is defined to be the dual of $W_{0}^{m, p}(\Omega), 1 / p+1 / q=1$, which is the completion of $C_{0}^{\infty}(\Omega)$ in $W^{m, p}(\Omega)$. Following the conventional notation, we write

$$
\nvdash_{\sigma}^{m}\left(R^{2}\right)=\left\{f \in W^{m, 2}\left(R^{2}\right)^{2}: \nabla \cdot f=0 \text { in } R^{2}\right\}
$$

with the obvious norm. We introduce

(1-1) $S=\left\{\phi \in C_{0}^{\infty}\left(R^{2}\right)^{2}: \nabla \cdot \phi=0\right.$ in $\left.R^{2}\right\}$;

$$
\xi_{p}=\left\{f \in W^{1,2}\left(R^{2}\right)^{2}: \frac{\partial f_{i}}{\partial x_{j}} \in L^{p}\left(R^{2}\right), i, j=1,2, \nabla \cdot f=0 \text { in } R^{2}\right\}
$$


with the norm

$$
\begin{gathered}
\|f\|_{\mathcal{F}_{p}}=\|f\|_{L^{2}\left(R^{2}\right)}+\|\nabla f\|_{L^{2}\left(R^{2}\right)}+\|\nabla f\|_{L^{p}\left(R^{2}\right)} \\
(1-3) \quad \mathcal{G}_{p}=\left\{f \in W^{1,2}\left(R^{2}\right)^{2}: \varepsilon_{i j}(f) \in L^{p}\left(R^{2}\right), i, j=1,2, \nabla \cdot f=0 \text { in } R^{2}\right\}
\end{gathered}
$$

with the norm

$$
\|f\|_{\mathcal{G}_{p}}=\|f\|_{L^{2}\left(R^{2}\right)}+\|\nabla f\|_{L^{2}\left(R^{2}\right)}+\sum_{i, j=1}^{2}\left\|\varepsilon_{i j}(f)\right\|_{L^{p}\left(R^{2}\right)} .
$$

It is apparent that $\mathcal{F}_{p}$ and $\mathcal{G}_{p}$ are Banach spaces for $1 \leq p \leq \infty$. For $m=$ nonnegative integer, we use the notation

$$
\begin{aligned}
H^{m}\left(E_{L}\right)=\left\{u: u=\sum_{k \in \mathbf{Z}^{2}} c_{k} e^{2 \pi i k \cdot x / L}, c_{k} \in \mathbf{C}^{2}, c_{k}=\right. & \overline{c_{-k}} \\
& \left.\sum_{k \in \mathbf{Z}^{2}}|k|^{2 m}\left|c_{k}\right|^{2}<\infty\right\}
\end{aligned}
$$

with the norm

$$
\begin{gathered}
\|u\|_{H^{m}\left(E_{L}\right)}=\left(\sum_{\alpha_{1}+\alpha_{2} \leq m} \int_{E_{L}}\left|\left(\frac{\partial}{\partial x_{1}}\right)^{\alpha_{1}}\left(\frac{\partial}{\partial x_{2}}\right)^{\alpha_{2}} u\right|^{2} d x\right)^{1 / 2} ; \\
J^{m}\left(E_{L}\right)=\left\{u \in H^{m}\left(E_{L}\right): \int_{E_{L}} u d x=0\right\}
\end{gathered}
$$

with the $H^{m}\left(E_{L}\right)$ norm;

$$
H_{\sigma}^{m}\left(E_{L}\right)=\left\{u \in H^{m}\left(E_{L}\right): \nabla \cdot u=0 \text { in } R^{2}\right\}
$$

with the $H^{m}\left(E_{L}\right)$ norm;

$$
J_{\sigma}^{m}\left(E_{L}\right)=\left\{u \in J^{m}\left(E_{L}\right): \nabla \cdot u=0 \text { in } R^{2}\right\}
$$

with the $H^{m}\left(E_{L}\right)$ norm;

$$
J_{\sigma}^{\infty}\left(E_{L}\right)=\bigcap_{m=1}^{\infty} J_{\sigma}^{m}\left(E_{L}\right)
$$

1.2. Some basic facts.

LEMMA 1.1. Let $m$ be a nonnegative integer. Then $J_{\sigma}^{m}\left(E_{L}\right)$ is characterized by

$$
J_{\sigma}^{m}\left(E_{L}\right)=\left\{u=\sum_{k \in \mathbf{Z}^{2}} c_{k} e^{2 \pi i k \cdot x / L} \in J^{m}\left(E_{L}\right): k \cdot c_{k}=0 \text { for each } k\right\} .
$$

This lemma is well known and the proof is omitted.

It is clear that $J_{\sigma}^{0}\left(E_{L}\right)$ is a closed subspace of $J^{0}\left(E_{L}\right)$. Let $P$ be the projection from $J^{0}\left(E_{L}\right)$ onto $J_{\sigma}^{0}\left(E_{L}\right)$. We can easily infer from Lemma 1.1 that the following lemma holds. 
LEMMA 1.2. For each $u=\sum_{k \in \mathbf{Z}^{2}} c_{k} e^{2 \pi i k \cdot x / L} \in J^{0}\left(E_{L}\right)$,

$$
P u=\sum_{k \in \mathbf{Z}^{2}}\left(c_{k}-k\left(c_{k} \cdot k\right) /|k|^{2}\right) e^{2 \pi i k \cdot x / L}
$$

and the orthogonal complement of $J_{\sigma}^{0}\left(E_{L}\right)$ in $J^{0}\left(E_{L}\right)$ is the set $\{v=\nabla q: q \in$ $\left.L^{2}\left(E_{L}\right), \nabla q \in J^{0}\left(E_{L}\right)\right\}$.

From the above lemmas, it is easily seen that for each $u \in J_{\sigma}^{2}\left(E_{L}\right), P \Delta u=\Delta u$ holds, i.e., the Stokes operator coincides with the Laplacian. We regard $\Delta$ as an operator in $J_{\sigma}^{0}\left(E_{L}\right)$ with the domain $J_{\sigma}^{2}\left(E_{L}\right)$. Then $\Delta^{-1}$ exists and is continuous from $J_{\sigma}^{0}\left(E_{L}\right)$ onto $J_{\sigma}^{2}\left(E_{L}\right)$; furthermore, it is a selfadjoint compact mapping in $J_{\sigma}^{0}\left(E_{L}\right)$. Consequently, there is a sequence of eigenfunctions which form an orthonormal basis of $J_{\sigma}^{0}\left(E_{L}\right)$ :

$$
\begin{gathered}
-\Delta \varphi_{j}=\lambda_{j} \varphi_{j}, \quad \varphi_{j} \in J_{\sigma}^{2}\left(E_{L}\right), j=1,2, \ldots, \\
0<\lambda_{1} \leq \lambda_{2} \leq, \ldots, \lambda_{j} \rightarrow \infty, \quad \text { as } j \rightarrow \infty .
\end{gathered}
$$

More precisely, $\left\{\varphi_{j}\right\}_{j=1}^{\infty}$ consists of

$$
\begin{aligned}
& \frac{\sqrt{2}}{L}\left(e_{\alpha}-\frac{k_{\alpha} k}{|k|^{2}}\right)\left|e_{\alpha}-\frac{k_{\alpha} k}{|k|^{2}}\right|^{-1} \cos \left(2 \pi k \cdot \frac{x}{L}\right) \\
& \frac{\sqrt{2}}{L}\left(e_{\alpha}-\frac{k_{\alpha} k}{|k|^{2}}\right)\left|e_{\alpha}-\frac{k_{\alpha} k}{|k|^{2}}\right|^{-1} \sin \left(2 \pi k \cdot \frac{x}{L}\right)
\end{aligned}
$$

and $\left\{\lambda_{j}\right\}_{j=1}^{\infty}$ consists of $4 \pi^{2}|k|^{2} / L^{2}$, where $k=\left(k_{1}, k_{2}\right) \in \mathbf{Z}^{2}, k \neq 0, \alpha=1,2$, and $e_{1}, e_{2}$ represents the canonical basis of $R^{2}$; see Temam [8] and Foias, Guillope and Temam [2].

LEMMA 1.3. $J_{\sigma}^{m}\left(E_{L}\right), m=$ nonnegative integer, can be characterized by

$$
J_{\sigma}^{m}\left(E_{L}\right)=\left\{u: u=\sum_{j=1}^{\infty} d_{j} \varphi_{j}, d_{j} \in R \text { and } \sum_{j=1}^{\infty} d_{j}^{2} \lambda_{j}^{m}<\infty\right\} .
$$

This is also well known and the proof is omitted.

The following estimates for functions of $J^{1}\left(E_{L}\right)$ will be used in the subsequent section.

LEMMA 1.4. For any $f \in J^{1}\left(E_{L}\right)$, it holds that $f \in L^{3}\left(E_{L}\right)^{2} \cap L^{4}\left(E_{L}\right)^{2}$ and

$$
\begin{aligned}
& \|f\|_{L^{3}\left(E_{L}\right)} \leq C\|f\|_{L^{2}\left(E_{L}\right)}^{2 / 3}\|\nabla f\|_{L^{2}\left(E_{L}\right)}^{1 / 3} \\
& \|f\|_{L^{4}\left(E_{L}\right)} \leq C\|f\|_{L^{2}\left(E_{L}\right)}^{1 / 2}\|\nabla f\|_{L^{2}\left(E_{L}\right)}^{1 / 2}
\end{aligned}
$$

and if $f \in J^{2}\left(E_{L}\right)$,

$$
\left\|\frac{\partial^{2} f}{\partial x_{i} \partial x_{j}}\right\|_{L^{2}\left(E_{L}\right)} \leq C\|\Delta f\|_{L^{2}\left(E_{L}\right)}, \quad i, j=1,2,
$$

where $C$ is a positive constant independent of $L$. 
ProOF. Let $f(x) \in C^{\infty}\left(E_{L}\right)^{2}$ such that $\int_{E_{L}} f(x) d x=0$. Define $g(y)=f(L y)$. Then $g(y) \in C^{\infty}\left(E_{1}(y)\right)^{2}$, and

$$
\begin{aligned}
& \|f(x)\|_{L^{4}\left(E_{L}\right)}^{4}=\int_{-L / 2}^{L / 2} \int_{-L / 2}^{L / 2}|f(x)|^{4} d x_{1} d x_{2} \\
& =L^{2} \int_{-1 / 2}^{1 / 2} \int_{-1 / 2}^{1 / 2}|f(L y)|^{4} d y_{1} d y_{2} \\
& =L^{2}\|g(y)\|_{L^{4}\left(E_{1}\right)}^{4}, \\
& \|f(x)\|_{L^{3}\left(E_{L}\right)}^{3}=L^{2}\|g(y)\|_{L^{3}\left(E_{1}\right)}^{3}, \\
& \|f(x)\|_{L^{2}\left(E_{L}\right)}^{2}=L^{2}\|g(y)\|_{L^{2}\left(E_{1}\right)}^{2}, \\
& \left\|\nabla_{x} f(x)\right\|_{L^{2}\left(E_{L}\right)}^{2}=\sum_{i, j=1}^{2} \int_{-L / 2}^{L / 2} \int_{-L / 2}^{L / 2}\left(\frac{\partial f_{i}\left(x_{1}, x_{2}\right)}{\partial x_{j}}\right)^{2} d x_{1} d x_{2} \\
& =\sum_{i, j=1}^{2} \int_{-1 / 2}^{1 / 2} \int_{-1 / 2}^{1 / 2}\left(\frac{\partial g_{i}\left(y_{1}, y_{2}\right)}{\partial y_{j}}\right)^{2} d y_{1} d y_{2} \\
& =\left\|\nabla_{y} g(y)\right\|_{L^{2}\left(E_{1}\right)}^{2} .
\end{aligned}
$$

By the Nirenberg-Garliardo inequality (see Theorem 10.1 of Friedman $[3]$ ), we have

$$
\begin{aligned}
\|g(y)\|_{L^{4}\left(E_{1}\right)} & \leq C\|g(y)\|_{W^{1,2}(\Omega)}^{1 / 2}\|g(y)\|_{L^{2}(\Omega)}^{1 / 2} \\
& \leq C\left\|\nabla_{y} g(y)\right\|_{L^{2}\left(E_{1}\right)}^{1 / 2}\|g(y)\|_{L^{2}\left(E_{1}\right)}
\end{aligned}
$$

where $\Omega$ is an open disc such that $E_{1} \subset \Omega \subset E_{2}$, and $C$ denotes positive constants independent of $g$ and $L$. We have used the fact that $g$ is 1-periodic and $\int_{E_{1}} g(y) d y=$ 0 . Similarly, we obtain

$$
\|g(y)\|_{L^{3}\left(E_{1}\right)} \leq C\left\|\nabla_{y} g(y)\right\|_{L^{2}\left(E_{1}\right)}^{1 / 3}\|g(y)\|_{L^{2}\left(E_{1}\right)}^{2 / 3}
$$

where $C$ is a positive constant independent of $g$ and $L$. Combining (1-18) through (1-23) we derive

$$
\begin{aligned}
& \|f(x)\|_{L^{4}\left(E_{L}\right)} \leq C\left\|\nabla_{x} f(x)\right\|_{L^{2}\left(E_{L}\right)}^{1 / 2}\|f(x)\|_{L^{2}\left(E_{L}\right)}^{1 / 2}, \\
& \|f(x)\|_{L^{3}\left(E_{L}\right)} \leq C\left\|\nabla_{x} f(x)\right\|_{L^{2}\left(E_{L}\right)}^{1 / 3}\|f(x)\|_{L^{2}\left(E_{L}\right)}^{2 / 3},
\end{aligned}
$$

where $C$ is a positive constant independent of $f$ and $L$. Using the standard technique of approximation, we arrive at (1-15) and (1-16) for any $f \in J^{1}\left(E_{L}\right)$. (1-17) follows from the series expansion of $f$.

Next we derive some useful properties of functions defined in $R^{2}$.

LEMMA 1.5. Let $1<p<\infty$. Suppose $v \in W^{1,2}\left(R^{2}\right)^{2}, \varepsilon_{i j}(v) \in L^{p}\left(R^{2}\right)$, for $i, j=1,2$. Then $\partial v_{i} / \partial x_{j} \in L^{p}\left(R^{2}\right)$ and it holds that

$$
\left\|\frac{\partial v_{i}}{\partial x_{j}}\right\|_{L^{p}\left(R^{2}\right)} \leq C \sum_{k, l=1}^{2}\left\|\varepsilon_{k l}(v)\right\|_{L^{p}\left(R^{2}\right)}, \quad \text { for } i, j=1,2
$$

where $C$ is a positive constant depending only on $p$. 
LEMMA 1.6. Let $1<p<\infty$. If $w \in L^{2}\left(R^{2}\right)$ and $\partial w / \partial x_{i}=\sum_{j=1}^{2}\left(\partial f_{i j} / \partial x_{j}\right)$, $i=1,2$, where $f_{i j} \in L^{p}\left(R^{2}\right), i, j=1,2$, then $w \in L^{p}\left(R^{2}\right)$ and

$$
\|w\|_{L^{p}\left(R^{2}\right)} \leq C \sum_{i, j=1}^{2}\left\|f_{i j}\right\|_{L^{p}\left(R^{2}\right)}
$$

where $C$ is a positive constant depending only on $p$.

PROOF. Recalling that the Riesz transform is a bounded linear operator from $L^{p}\left(R^{2}\right)$ into itself for $1<p<\infty$, we can find a function $w^{*}$ in $L^{p}\left(R^{2}\right)$ such that

$$
\begin{aligned}
\Delta w^{*} & =\sum_{i, j=1}^{2} \frac{\partial^{2} f_{i j}}{\partial x_{i} \partial x_{j}}, \\
\left\|w^{*}\right\|_{L^{p}\left(R^{2}\right)} & \leq C \sum_{i, j=1}^{2}\left\|f_{i j}\right\|_{L^{p}\left(R^{2}\right)},
\end{aligned}
$$

where $C$ is a positive constant depending only on $p$. Now let us set $g=w-w^{*}$. Then $g \in L^{2}+L^{p}$ and $\Delta g=0$ holds. Hence, $g$ is a harmonic function, and it holds that

$$
\left|\int_{R^{2}} g \psi d x\right| \leq C\left\{\|\psi\|_{L^{2}\left(R^{2}\right)}+\|\psi\|_{L^{q}\left(R^{2}\right)}\right\}
$$

for any $\psi \in L^{2}\left(R^{2}\right) \cap L^{q}\left(R^{2}\right), 1 / p+1 / q=1$, where $C$ is a positive constant independent of $\psi$. Define

$$
\psi_{\alpha}(x)= \begin{cases}1 & \text { for }|x-\alpha| \leq 1 \\ 0 & \text { for }|x-\alpha|>1\end{cases}
$$

Then by the mean value theorem,

$$
\int_{R^{2}} g \psi_{\alpha} d x=\pi g(\alpha)
$$

and consequently, (1-30) implies that $g(\alpha)$ is uniformly bounded for all $\alpha \in R^{2}$. Thus, $g(x) \equiv$ constant for all $x \in R^{2}$. In the meantime, there is a sequence of nonnegative functions $\left\{\xi_{m}(x)\right\}_{m=1}^{\infty}$ such that $\left\|\xi_{m}\right\|_{L^{2}\left(R^{2}\right)}+\left\|\xi_{m}\right\|_{L^{q}\left(R^{2}\right)} \leq M<\infty$ for all $m$ and $\left\|\xi_{m}\right\|_{L^{1}\left(R^{2}\right)}$ tends to infinity as $m \rightarrow \infty$. From (1-30), we conclude that $g \equiv 0$.

PROOF OF LEMMA 1.5. Borrowing an idea from Duvaut and Lions [1], we make use of the identity

$$
\frac{\partial^{2} u_{i}}{\partial x_{j} \partial x_{k}}=\frac{\partial}{\partial x_{j}} \varepsilon_{i k}(u)+\frac{\partial}{\partial x_{k}} \varepsilon_{i j}(u)-\frac{\partial}{\partial x_{i}} \varepsilon_{j k}(u)
$$

and Lemma 1.6 to prove the assertion.

LEMMA 1.7. $\xi_{p}=\mathcal{G}_{p}$, for $1<p<\infty$.

PROOF. Obvious from Lemma 1.5.

We next define

$$
\tilde{\xi}_{p}=\left\{f \in W^{1,2}\left(R^{2}\right)^{2}: \frac{\partial f_{i}}{\partial x_{j}} \in L^{p}\left(R^{2}\right), i, j=1,2\right\} \quad \text { for } 1 \leq p<\infty,
$$

equipped with the same norm as $\mathcal{F}_{p}$. Then, we have the following lemma. 
LEMMA 1.8. $C_{0}^{\infty}\left(R^{2}\right)^{2}$ is dense in $\tilde{F}_{p}, 1 \leq p<\infty$.

PROOF. Let $f \in \tilde{f}_{p}$ and define

$$
\psi_{r}(x)= \begin{cases}1 & \text { for }|x| \leq r \\ 2-|x| / r & \text { for } r \leq|x| \leq 2 r \\ 0 & \text { for } 2 r \leq|x|\end{cases}
$$

Put $f_{r}(x)=f(x) \psi_{r}(x)$. Then, $\left|\nabla f-\nabla f_{r}\right|=0$ for $|x|<r$, and

$$
\begin{aligned}
\int_{r \leq|x|}\left|\nabla f_{r}\right|^{p} d x & \leq C_{p}\left\{\int_{r \leq|x| \leq 2 r}|\nabla f|^{p} d x+\int_{r \leq|x| \leq 2 r}|f|^{p} \frac{1}{r^{p}} d x\right\} \\
& \leq C_{p}\left\{\int_{r \leq|x| \leq 2 r}|\nabla f|^{p} d x+\frac{\sqrt{3 \pi}}{r^{p-1}}\left(\int_{r \leq|x|}|f|^{2 p} d x\right)^{1 / 2}\right\},
\end{aligned}
$$

where $C_{p}$ is a positive constant depending only on $1 \leq p<\infty$. We notice that $|f| \in L^{q}\left(R^{2}\right)$, for any $2 \leq q<\infty$, since $f \in W^{1,2}\left(R^{2}\right)^{2}$. Now it is evident from (1-36) that

$$
\int_{R^{2}}\left|f-f_{r}\right|^{2} d x+\int_{R^{2}}\left|\nabla f-\nabla f_{r}\right|^{2} d x+\int_{R^{2}}\left|\nabla f-\nabla f_{r}\right|^{p} d x \rightarrow 0 \quad \text { as } r \rightarrow \infty
$$

In the meantime, using the Friedrichs mollifier, we can approximate each $f_{r}(x)$ by a sequence of functions in $C_{0}^{\infty}\left(R^{2}\right)^{2}$ with respect to the $\tilde{\mathcal{F}}_{p}$ norm, which proves the assertion of the lemma.

LEMMA 1.9. $S$ is dense in $\mathcal{F}_{p}$ for $1<p<\infty$.

ProOF. By Lemma 1.8, each element of $\tilde{\mathcal{F}}_{p}^{*}$ (the dual of $\tilde{f}_{p}$ ) can be identified as an element of $D^{\prime}\left(R^{2}\right)^{2}$. In the meantime, each element of $\mathcal{F}_{p}^{*}$ (the dual of $\mathcal{F}_{p}$ ) can be represented by an element of $\tilde{F}_{p}^{*}$ by the Hahn-Banach theorem. Assume that $S$ is not dense in $\mathcal{F}_{p}$. Then, there is $\Lambda \in \tilde{\mathcal{F}}_{p}^{*}$ which annihilates $S$, but $\Lambda f \neq 0$ for some $f \in \mathcal{F}_{p}$. Since $\Lambda$ is a distribution annihilating $S$, we can find a distribution $\Phi$ such that $\Lambda=\nabla \Phi$ by simple application of Theorem VI in Schwartz [6]. Using the same argument that is used to derive a repesentation formula for $W^{-m, p}\left(R^{2}\right)$, we see that $\Lambda=\left(\Lambda_{1}, \Lambda_{2}\right)$ can be expressed as

$$
\Lambda_{i}=\frac{\partial \Phi}{\partial x_{i}}=\xi_{i}+\sum_{j=1}^{2} \frac{\partial \eta_{i j}}{\partial x_{j}}+\sum_{j=1}^{2} \frac{\partial \rho_{i j}}{\partial x_{j}}, \quad i=1,2,
$$

for some $\xi_{i}, \eta_{i j} \in L^{2}\left(R^{2}\right)$ and $\rho_{i j} \in L^{q}\left(R^{2}\right), 1 / q+1 / p=1, i, j=1,2$. Now, with the aid of the Riesz transform, we can find $\alpha(x) \in L^{2}\left(R^{2}\right), \beta(x) \in L^{q}\left(R^{2}\right)$, $\gamma_{i}(x) \in L^{2}\left(R^{2}\right), i=1,2$, such that

$$
\begin{gathered}
\Delta \alpha=\sum_{i, j=1}^{2} \frac{\partial^{2} \eta_{i j}}{\partial x_{i} \partial x_{j}}, \\
\Delta \beta=\sum_{i, j=1}^{2} \frac{\partial^{2} \rho_{i j}}{\partial x_{i} \partial x_{j}}, \\
\Delta \gamma_{i}=\sum_{j=1}^{2} \frac{\partial^{2} \xi_{j}}{\partial x_{i} \partial x_{j}}, \quad i=1,2 .
\end{gathered}
$$


From the relation between the Fourier transforms of $\gamma_{i}$ 's and $\xi_{j}$ 's, it is clear that $\partial \gamma_{i} / \partial x_{j}=\partial \gamma_{j} / \partial x_{i}$ holds, $i, j=1,2$, which implies the existence of a distribution $\theta$ such that

$$
\gamma_{i}=\partial \theta / \partial x_{i}, \quad i=1,2,
$$

on account of Theorem VI in Schwartz [6]. Next we observe that (1-38) yields

$$
\Delta \Phi=\sum_{i=1}^{2} \frac{\partial \xi_{i}}{\partial x_{i}}+\sum_{i, j=1}^{2} \frac{\partial^{2} \eta_{i j}}{\partial x_{i} \partial x_{j}}+\sum_{i, j=1}^{2} \frac{\partial^{2} \rho_{i j}}{\partial x_{i} \partial x_{j}}
$$

and thus, in conjunction with (1-39) to (1-42),

$$
\Delta \frac{\partial \Phi}{\partial x_{k}}=\Delta \frac{\partial \theta}{\partial x_{k}}+\Delta \frac{\partial \alpha}{\partial x_{k}}+\Delta \frac{\partial \beta}{\partial x_{k}}, \quad k=1,2
$$

We write

$$
g=\nabla \Phi-\nabla \theta-\nabla \alpha-\nabla \beta
$$

Then, by (1-44), $g$ is an $R^{2}$-valued harmonic function, which also belongs to $L^{2}\left(R^{2}\right)^{2}+W^{-1,2}\left(R^{2}\right)^{2}+W^{-1, q}\left(R^{2}\right)^{2}$.

Let us introduce

$$
\psi_{\alpha}(x)= \begin{cases}1, & \text { for }|x-\alpha| \leq 1 \\ 2-|x-\alpha|, & \text { for } 1 \leq|x-\alpha| \leq 2 \\ 0, & \text { for } 2 \leq|x-\alpha|\end{cases}
$$

Then, $\psi_{\alpha}(x) \in L^{2}\left(R^{2}\right) \cap W^{1,2}\left(R^{2}\right) \cap W^{1, p}\left(R^{2}\right)$, for each $\alpha \in R^{2}$, and, by the mean value theorem,

$$
\int_{R^{2}} g \psi_{\alpha} d x=\frac{7}{3} \pi g(\alpha)
$$

Since $g \in L^{2}\left(R^{2}\right)^{2}+W^{-1,2}\left(R^{2}\right)^{2}+W^{-1, q}\left(R^{2}\right)^{2}$, it holds that

$$
\left|\int_{R^{2}} g \psi_{\alpha} d x\right| \leq M\left\{\left\|\psi_{\alpha}\right\|_{L^{2}\left(R^{2}\right)}+\left\|\psi_{\alpha}\right\|_{W^{1,2}\left(R^{2}\right)}+\left\|\psi_{\alpha}\right\|_{W^{1, p}\left(R^{2}\right)}\right\}
$$

for a positive constant $M$ independent of $\alpha$. Consequently, $g(\alpha)$ is uniformly bounded for all $\alpha \in R^{2}$, which implies $g(x)=\left(C_{1}, C_{2}\right)$ for all $x \in R^{2}$, for some constant vector $\left(C_{1}, C_{2}\right) \in R^{2}$. Now we choose $h(x) \in C_{0}^{\infty}\left(R^{2}\right)$ such that $\int_{R^{2}} h d x>0$, and define $h_{n}(x)=h\left(x_{1}+n, x_{2}+n\right)$. Then, for each $n$, we see from (1-45) that

$$
\begin{aligned}
\int_{R^{2}} C_{i} h_{n} d x= & \int_{R^{2}} \xi_{i} h_{n} d x-\sum_{j=1}^{2} \int_{R^{2}} \eta_{i j} \frac{\partial h_{n}}{\partial x_{j}} d x-\sum_{j=1}^{2} \rho_{i j} \frac{\partial h_{n}}{\partial x_{j}} d x \\
& +\int_{R^{2}} \alpha \frac{\partial h_{n}}{\partial x_{i}} d x+\int_{R^{2}} \beta \frac{\partial h_{n}}{\partial x_{i}} d x-\int_{R^{2}} \frac{\partial \theta}{\partial x_{i}} h_{n} d x
\end{aligned}
$$

Since $\xi_{i}, \eta_{i j}, \alpha, \partial \theta / \partial x_{i} \in L^{2}\left(R^{2}\right)$ and $\rho_{i j}, \beta \in L^{q}\left(R^{2}\right), i, j=1,2$, the right side of (1-49) tends to zero as $n \rightarrow \infty$, while the left side does not change as $n \rightarrow \infty$. Hence, we conclude $C_{i}=0, i=1,2$, and

$$
\nabla \Phi=\nabla \alpha+\nabla \beta+\nabla \theta
$$


from which it follows that for every $f=\left(f_{1}, f_{2}\right) \in \mathcal{F}_{p}$,

$$
-\Lambda f=\sum_{i=1}^{2} \int_{R^{2}} \alpha \frac{\partial f_{i}}{\partial x_{i}} d x+\sum_{i=1}^{2} \int_{R^{2}} \beta \frac{\partial f_{i}}{\partial x_{i}} d x-\sum_{i=1}^{2} \int_{R^{2}} \frac{\partial \theta}{\partial x_{i}} f_{i} d x
$$

holds. Since $f \in W^{1,2}\left(R^{2}\right)^{2}$ and $\nabla \cdot f=0$ in the sense of distribution in $R^{2}$, it is evident that $\nabla \cdot f=0$ for almost all $x \in R^{2}$ and thus, the first two terms on the right side of (1-51) are zero. At the same time, there is a sequence $\left\{H_{n}(x)\right\}_{n=1}^{\infty}$ in $S$ such that $H_{n}$ converges to $f$ in the $L^{2}\left(R^{2}\right)^{2}$ norm. Therefore,

$$
\begin{aligned}
\sum_{i=1}^{2} \int_{R^{2}} \frac{\partial \theta}{\partial x_{i}} f_{i} d x & =\lim _{n \rightarrow \infty} \int_{R^{2}} \nabla \theta \cdot H_{n} d x=\lim _{n \rightarrow \infty}\left\langle\nabla \theta, H_{n}\right\rangle \\
& =-\lim _{n \rightarrow \infty}\left\langle\theta, \nabla \cdot H_{n}\right\rangle=0
\end{aligned}
$$

where $\langle$,$\rangle stands for the duality pairing between C_{0}^{\infty}\left(R^{2}\right)^{2}$ and $D^{\prime}\left(R^{2}\right)^{2}$. Therefore, $\Lambda f=0$, which contradicts the assumption that $S$ is not dense in $\mathcal{F}_{p}$.

In conjunction with the above lemma, we state

LEMMA 1.10. $\mathcal{G}_{1}$ is separable.

PROOF. Consider a bounded linear operator $\Gamma$ from $\mathcal{G}_{1}$ into $W^{1,2}\left(R^{2}\right)^{2} \times$ $L^{1}\left(R^{2}\right)^{3}$ :

$$
f \rightarrow\left(f, \varepsilon_{11}(f), \varepsilon_{12}(f), \varepsilon_{22}(f)\right) \text {. }
$$

Then, $\Gamma\left(G_{1}\right)$ is a closed subspace of $W^{1,2}\left(R^{2}\right)^{2} \times L^{1}\left(R^{2}\right)^{3}$ and $\Gamma$ is a topological isomorphism from $\mathcal{G}_{1}$ onto $\Gamma\left(\mathcal{G}_{1}\right)$. Since $W^{1,2}\left(R^{2}\right)^{2} \times L^{1}\left(R^{2}\right)^{3}$ is separable, $\mathcal{G}_{1}$ as well as $\Gamma\left(\mathcal{G}_{1}\right)$ is separable.

\section{Existence and uniqueness of strong solution.}

DEFINITION 2.1. $u(x, t)$ is called a strong solution on $[0, T]$ of $(0-1)$ to $(0-3)$ if $u \in L^{2}\left(0, T ; \nvdash_{\sigma}^{2}\left(R^{2}\right)\right) \cap L^{2}\left(0, T ; \mathcal{G}_{1}\right), \partial u / \partial t \in L^{2}\left(0, T ; \nvdash_{\sigma}^{0}\left(R^{2}\right)\right), u(x, 0)=u_{0}(x)$, and for each $w \in \mathcal{G}_{1}$,

$$
\begin{aligned}
& \left\langle\frac{\partial u}{\partial t}, w-u\right\rangle+\langle(u \cdot \nabla) u, w-u\rangle+\mu a(u, w-u) \\
& \quad+\nu \int_{R^{2}} D(w) d x-\nu \int_{R^{2}} D(u) d x \geq\langle F, w-u\rangle
\end{aligned}
$$

holds for almost all $t \in[0, T]$, where $\langle$,$\rangle denotes the inner product in L^{2}\left(R^{2}\right)^{2}$.

Let $\mathcal{G}_{1,0}$ be the completion of $S$ with respect to the $\mathcal{G}_{1}$ norm. Our main result is

THEOREM 2.2. Let $u_{0}(x) \in \mathcal{G}_{1,0}$ and $F(x, t) \in L^{2}\left(0, T ; L^{2}\left(R^{2}\right)^{2}\right)$. Then, there is a unique strong solution $u(x, t)$ of (0-1) to (0-3) and furthermore, $u(x, t) \in$ $L^{\infty}\left(0, T ; \mathcal{G}_{1}\right)$. 
We shall sketch the procedure to prove the above theorem. It is convenient to introduce the following notations:

$$
\begin{gathered}
j_{L, \eta}(v)=\frac{1}{1+\eta} \int_{E_{L}} D(v)^{1+\eta} d x \\
j_{\eta}(v)=\frac{1}{1+\eta} \int_{R^{2}} D(v)^{1+\eta} d x \\
j(v)=\int_{R^{2}} D(v) d x .
\end{gathered}
$$

Step I. Regularize (2-1) by substituting $j_{L, \eta}(\cdot)$ for $j(\cdot)$. We employ the Galerkin method, and use the convexity and differentiability of $j_{L, \eta}(\cdot)$ to establish the existence of a strong and spatially $L$-periodic solution to this regularized problem. We also obtain enough a priori estimates independent of $L$.

Step II. With the aid of the uniform a priori estimates obtained in Step I, we pass the periodic solutions to a limit by expanding $E_{L}$ to the whole space. It is then shown that this limit function is a strong solution of $(2-1)$ with $j(\cdot)$ replaced by $j_{\eta}(\cdot)$.

Step III. We finally let $\eta \rightarrow 0$ and arrive at a strong solution of (2-1). The uniqueness of solution is also proved.

2.1. The Cauchy problem with periodic boundary condition. Let $v_{0}(x) \in J_{\sigma}^{2}\left(E_{L}\right)$ and $F_{L}(x, t) \in C\left([0, T] ; J^{0}\left(E_{L}\right)\right), 0<T<\infty$, satisfying

$$
\begin{gathered}
\left\|v_{0}\right\|_{J_{\sigma}^{1}\left(E_{L}\right)}+\left\|D\left(v_{0}\right)\right\|_{L^{1}\left(E_{L}\right)} \leq K, \\
\left\|F_{L}\right\|_{L^{2}\left(0, T ; J^{0}\left(E_{L}\right)\right)} \leq K
\end{gathered}
$$

for some positive constant $K$. Then, we state

Proposition 2.3. Fix any $0<\eta<1$. There is a function $v(x, t)$ such that

(i) $v \in C\left([0, T] ; J_{\sigma}^{1}\left(E_{L}\right)\right) \cap L^{2}\left(0, T ; J_{\sigma}^{2}\left(E_{L}\right)\right), \partial v / \partial t \in L^{2}\left(0, T ; J_{\sigma}^{0}\left(E_{L}\right)\right), v(x, 0)$ $=v_{0}(x), D(v)^{1+\eta} \in L^{\infty}\left(0, T ; L^{1}\left(E_{L}\right)\right)$;

(ii) for each $g \in L^{2}\left(0, T ; H_{\sigma}^{1}\left(E_{L}\right)\right)$,

$$
\begin{aligned}
& \int_{0}^{T}\left\langle\frac{\partial v}{\partial t}, g\right\rangle d t+\int_{0}^{T}\langle(v \cdot \nabla) v, g\rangle d t-\mu \int_{0}^{T}\langle\Delta v, g\rangle d t \\
& \quad+\nu \int_{0}^{T} j_{L, \eta}(v+g) d t-\nu \int_{0}^{T} j_{L, \eta}(v) d t \geq \int_{0}^{T}\left\langle F_{L}, g\right\rangle d t
\end{aligned}
$$

where $\langle$,$\rangle denotes the inner product in L\left(E_{L}\right)^{2}$;

(iii) it holds that

$$
\begin{array}{lc}
(2-8) & \|v\|_{C\left([0, T] ; J_{\sigma}^{1}\left(E_{L}\right)\right)} \leq M(\mu, K), \\
(2-9) & \|v\|_{L^{2}\left(0, T ; J_{\sigma}^{2}\left(E_{L}\right)\right)} \leq M(\mu, K), \\
(2-10) & \|\partial v / \partial t\|_{L^{2}\left(0, T ; J_{\sigma}^{0}\left(E_{L}\right)\right)} \leq \nu M(K)+M(\mu, K), \\
(2-11) \nu\left\|D(v)^{1+\eta}\right\|_{L^{1}\left(E_{L}\right)} \leq \nu M(K)+M(\mu, K), & \text { for almost all } t \in[0, T],
\end{array}
$$

where $M(K)$ is a positive constant depending only on $K$ while $M(\mu, K)$ denotes positive constants depending only on $\mu$ and $K$, and independent of $\nu, \eta$ and the period $L$.

Before proving this proposition, we list some useful properties of $j_{L, \eta}(\cdot)$ defined by $(2-2)$. 
LEMMA 2.4. (i) For every $f, g \in H^{1}\left(E_{L}\right)$, it holds that

$$
\left(j_{L, \eta}^{\prime}(f), g\right)=\sum_{i, j=1}^{2} 2 \int_{E_{L}} D(f)^{-1+\eta} \varepsilon_{i j}(f) \varepsilon_{i j}(g) d x
$$

and

$$
j_{L, \eta}(f+g)-j_{L, \eta}(f) \geq\left(j_{L, \eta}^{\prime}(f), g\right)
$$

where $j_{L, \eta}^{\prime}(f)$ denotes the Gateaux differential of $j_{L, \eta}(\cdot)$ at $f$ and $(, \quad)$ stands for the duality pairing between $H^{1}\left(E_{L}\right)$ and its dual.

(ii) For each $f \in H^{3}\left(E_{L}\right)$, we have

$$
\left(j_{L, \eta}^{\prime}(f), \frac{\partial^{2}}{\partial x_{i}^{2}} f\right) \leq 0, \quad i=1,2 .
$$

PROOF. (2-12) and (2-13) are well known; see Duvaut and Lions [1]. To prove $(2-14)$ for $i=1$, we put

$$
f_{h}\left(x_{1}, x_{2}\right)=f\left(x_{1}+h, x_{2}\right),
$$

and notice that as $h \rightarrow 0+$,

$$
\frac{f_{h}-2 f+f_{-h}}{h^{2}} \rightarrow \frac{\partial^{2}}{\partial x_{1}^{2}} f \text { in the } H^{1}\left(E_{L}\right) \text { norm }
$$

and that

$$
\left(j_{L, \eta}^{\prime}(f), f_{h}-2 f+f_{-h}\right)=-\left(j_{L, \eta}^{\prime}\left(f_{h}\right)-j_{L, \eta}^{\prime}(f), f_{h}-f\right),
$$

where the periodicity of $f$ has been used. Now the right-hand side of $(2-17)$ is nonpositive since $j_{L, \eta}^{\prime}(\cdot)$ is monotone, which follows from the convexity of $j_{L, \eta}(\cdot)$. Hence, (2-14) is true for $i=1$. The same argument applies for the case $i=2$.

PROOF OF PROPOSITION 2.3. Put $v_{m}(x, t)=\sum_{k=1}^{m} \alpha_{m k}(t) \varphi_{k}(x)$, where $\left\{\varphi_{k}\right\}_{k=1}^{\infty}$ is the sequence of eigenfunctions in (1-10). We determine

$$
\alpha_{m k}(t) \in C^{1}\left(\left[0, T_{m}\right]\right), \quad k=1, \ldots, m,
$$

so that

$$
\begin{aligned}
\left\langle\partial v_{m} / \partial t, \varphi_{l}\right\rangle & +\left\langle\left(v_{m} \cdot \nabla\right) v_{m}, \varphi_{l}\right\rangle-\mu\left\langle\Delta v_{m}, \varphi_{l}\right\rangle \\
& +\nu\left(j_{L, \eta}^{\prime}\left(v_{m}\right), \varphi_{l}\right)=\left\langle F_{L}, \varphi_{l}\right\rangle, \quad l=1, \ldots, m,
\end{aligned}
$$

and

$$
\alpha_{m k}(0)=\alpha_{k}=\left\langle v_{0}, \varphi_{k}\right\rangle, \quad k=1, \ldots, m,
$$

where $\langle$,$\rangle denotes the inner product in L^{2}\left(E_{L}\right)^{2}$ and $($,$) stands for the duality$ pairing between $H^{1}\left(E_{L}\right)$ and its dual; see (2-12). By the general theory of ordinary differential equations, there is a solution $\alpha_{m k}(t) \in C^{1}\left(\left[0, T_{m}\right]\right), k=1, \ldots, m$, for some $0<T_{m} \leq T$. It follows from $(2-18)$ that

$$
\left\langle\partial v_{m} / \partial t, v_{m}\right\rangle-\mu\left\langle\Delta v_{m}, v_{m}\right\rangle \leq\left\langle F_{L}, v_{m}\right\rangle
$$

and thus,

$$
\begin{aligned}
& \frac{1}{2} \frac{d}{d t} \int_{E_{L}}\left|v_{m}(x, t)\right|^{2} d x+\mu \int_{E_{L}}\left|\nabla v_{m}(x, t)\right|^{2} d x \\
& \quad \leq \frac{1}{2} \int_{E_{L}}\left|v_{m}(x, t)\right|^{2} d x+\frac{1}{2} \int_{E_{L}}\left|F_{L}(x, t)\right|^{2} d x .
\end{aligned}
$$


Combing (2-5), (2-6) and (2-21), we obtain

$$
\int_{E_{L}}\left|v_{m}(x, t)\right|^{2} d x \leq M(K) \text { for all } t \in\left[0, T_{m}\right]
$$

where $M(K)$ is a positive constant depending only on $K$ and independent of $T_{m}$. Hence, we may set $T_{m}=T$ and derive

$$
\mu \int_{0}^{T} \int_{E_{L}}\left|\nabla v_{m}(x, t)\right|^{2} d x d t \leq M(K)
$$

where $M(K)$ depends only on $K$. Next we substitute $\sum_{k=1}^{m} a_{m k}(t) \lambda_{k} \varphi_{k}(x)$, which is equal to $-\Delta v_{m}$, for $\varphi_{l}$ in (2-18) and derive, using (2-14),

$$
\begin{aligned}
\frac{1}{2} \frac{d}{d t} \int_{E_{L}}\left|\nabla v_{m}\right|^{2} d x & +\mu \int_{E_{L}}\left|\Delta v_{m}\right|^{2} d x \\
\leq & \left\|\left(v_{m} \cdot \nabla\right) v_{m}\right\|_{L^{2}\left(E_{L}\right)}\left\|\Delta v_{m}\right\|_{L^{2}\left(E_{L}\right)} \\
& +\left\|F_{L}\right\|_{L^{2}\left(E_{L}\right)}\left\|\Delta v_{m}\right\|_{L^{2}\left(E_{L}\right)} .
\end{aligned}
$$

In the meantime, by virtue of Lemma 1.4 , we get

$$
\begin{aligned}
\left\|\left(v_{m} \cdot \nabla\right) v_{m}\right\|_{L^{2}\left(E_{L}\right)} & \leq C\left\|v_{m}\right\|_{L^{4}\left(E_{L}\right)}\left\|\nabla v_{m}\right\|_{L^{4}\left(E_{L}\right)} \\
& \leq C\left\|v_{m}\right\|_{L^{2}\left(E_{L}\right)}^{1 / 2}\left\|\nabla v_{m}\right\|_{L^{2}\left(E_{L}\right)}\left\|\Delta v_{m}\right\|_{L^{2}\left(E_{L}\right)}^{1 / 2}
\end{aligned}
$$

and consequently, by Young's inequality,

$$
\begin{aligned}
& \left\|\left(v_{m} \cdot \nabla\right) v_{m}\right\|_{L^{2}\left(E_{L}\right)}\left\|\Delta v_{m}\right\|_{L^{2}\left(E_{L}\right)} \\
& \quad \leq \frac{\mu}{4}\left\|\Delta v_{m}\right\|_{L^{2}\left(E_{L}\right)}^{2}+\frac{C}{\mu^{3}}\left\|v_{m}\right\|_{L^{2}\left(E_{L}\right)}^{2}\left\|\nabla v_{m}\right\|_{L^{2}\left(E_{L}\right)}^{4},
\end{aligned}
$$

where $C$ stands for positive constants independent of $m, \mu, \nu, \eta$ and the period $L$. Now (2-24) and (2-26) yield

$$
\begin{aligned}
& \frac{1}{2} \frac{d}{d t} \int_{E_{L}}\left|\nabla v_{m}\right|^{2} d x+\frac{\mu}{2} \int_{E_{L}}\left|\Delta v_{m}\right|^{2} d x \\
& \quad \leq \frac{C}{\mu^{3}}\left\|v_{m}\right\|_{L^{2}\left(E_{L}\right)}^{2}\left\|\nabla v_{m}\right\|_{L^{2}\left(E_{L}\right)}^{4}+\frac{1}{\mu}\left\|F_{L}\right\|_{L^{2}\left(E_{L}\right)}^{2} .
\end{aligned}
$$

Borrowing an idea from Temam [7], we set $\sigma(t)=\left\|v_{m}\right\|_{L^{2}\left(E_{L}\right)}^{2}\left\|\nabla v_{m}\right\|_{L^{2}\left(E_{L}\right)}^{2}$. Then, by $(2-22)$ and $(2-23)$,

$$
\int_{0}^{T} \sigma(t) d t \leq M(\mu, K)
$$

holds where $M(\mu, K)$ is a positive constant independent of $m, \nu, \eta$ and $L$; and we rewrite $(2-27)$,

$$
\frac{1}{2} \frac{d}{d t} \int_{E_{L}}\left|\nabla v_{m}\right|^{2} d x \leq \frac{C}{\mu^{3}} \sigma(t) \int_{E_{L}}\left|\nabla v_{m}\right|^{2} d x+\frac{1}{\mu}\left\|F_{L}\right\|_{L^{2}\left(E_{L}\right)}^{2} .
$$

Using the Gronwall inequality, we derive

$$
\int_{E_{L}}\left|\nabla v_{m}\right|^{2} d x \leq M(\mu, K), \quad \text { for all } t \in[0, T]
$$


which, together with (2-27), implies

$$
\int_{0}^{T} \int_{E_{L}}\left|\Delta v_{m}\right|^{2} d x d t \leq M(\mu, K)
$$

where $M(\mu, K)$ is a positive constant independent of $m, \nu, \eta$ and $L$. Next we substitute $(\partial / \partial t) \sum_{k=1}^{m} a_{m k}(t) \varphi_{k}(x)$ for $\varphi_{l}$ in $(2-18)$ :

$$
\begin{aligned}
& \int_{E_{L}}\left|\frac{\partial v_{m}}{\partial t}\right|^{2} d x+\frac{\mu}{2} \frac{d}{d t} \int_{E_{L}}\left|\nabla v_{m}\right|^{2} d x+\nu \frac{d}{d t} j_{L, \eta}\left(v_{m}\right) \\
& \quad \leq\left\|\left(v_{m} \cdot \nabla\right) v_{m}\right\|_{L^{2}\left(E_{L}\right)}\left\|\frac{\partial v_{m}}{\partial t}\right\|_{L^{2}\left(E_{L}\right)}+\left\|F_{L}\right\|_{L^{2}\left(E_{L}\right)}\left\|\frac{\partial v_{m}}{\partial t}\right\|_{L^{2}\left(E_{L}\right)},
\end{aligned}
$$

using (2-25),

$\leq \frac{1}{2} \int_{E_{L}}\left|\frac{\partial v_{m}}{\partial t}\right|^{2} d x+\left\|F_{L}\right\|_{L^{2}\left(E_{L}\right)}^{2}+C \int_{E_{L}}\left|\Delta v_{m}\right|^{2} d x+C\left\|v_{m}\right\|_{L^{2}\left(E_{L}\right)}^{2}\left\|\nabla v_{m}\right\|_{L^{2}\left(E_{L}\right)}^{4}$ where $C$ is a positive constant independent of $m, \nu, \eta$ and $L$, from which it follows by using (2-22), (2-30) and (2-31) that

$$
\begin{gathered}
\frac{1}{2} \int_{0}^{t} \int_{E_{L}}\left|\frac{\partial v_{m}}{\partial t}\right|^{2} d x d t+\frac{\mu}{2} \int_{E_{L}}\left|\nabla v_{m}(x, t)\right|^{2} d x+\nu j_{L, \eta}\left(v_{m}(x, t)\right) \\
\leq \frac{\mu}{2} \int_{E_{L}}\left|\nabla v_{m}(x, 0)\right|^{2} d x+\nu j_{L, \eta}\left(v_{m}(x, 0)\right)+M(\mu, K)
\end{gathered}
$$

for all $t \in[0, T]$, where $M(\mu, K)$ is a positive constant independent of $m, \nu, \eta$ and $L$. It follows from Lemma 1.3 and (2-19) that $v_{m}(x, 0) \rightarrow v_{0}(x)$ strongly in $J_{\sigma}^{1}\left(E_{L}\right)$ and hence, $D\left(v_{m}(x, 0)\right) \rightarrow D\left(v_{0}\right)$ strongly in $L^{1}\left(E_{L}\right)$ as $m \rightarrow \infty$. Combining this and (2-5), we find that there is a positive integer $N$ such that for all $m \geq N$,

$$
\left\|v_{m}(x, 0)\right\|_{J_{\sigma}^{1}\left(E_{L}\right)}+\left\|D\left(v_{m}(x, 0)\right)\right\|_{L^{1}\left(E_{L}\right)} \leq 2 K
$$

and consequently, by Hölder's inequality,

$$
\begin{aligned}
& \left\|D\left(v_{m}(x, 0)\right)\right\|_{L^{1+\eta}\left(E_{L}\right)} \\
& \quad \leq\left\|D\left(v_{m}(x, 0)\right)\right\|_{L^{2}\left(E_{L}\right)}^{2 \eta /(1+\eta)}\left\|D\left(v_{m}(x, 0)\right)\right\|_{L^{1}\left(E_{L}\right)}^{(1-\eta) /(1+\eta)} \leq M(K) .
\end{aligned}
$$

Combining (2-33) to (2-35), we deduce that for all $m \geq N$,

$$
\int_{0}^{T} \int_{E_{L}}\left|\frac{\partial v_{m}}{\partial t}\right|^{2} d x d t \leq M(\mu, K)+\nu M(K)
$$

and, for all $t \in[0, T]$,

$$
\nu j_{L, \eta}\left(v_{m}(x, t)\right) \leq M(\mu, K)+\nu M(K)
$$

where $M(\mu, K)$ and $M(K)$ are positive constants independent of $m, \nu, \eta$ and $L$. By virtue of (2-22), (2-30), (2-31) and (2-36), we can extract a subsequence still denoted by $\left\{v_{m}\right\}$ such that as $m \rightarrow \infty$,

$$
\begin{aligned}
v_{m} & \rightarrow v \quad \text { weakly in } L^{2}\left(0, T ; J_{\sigma}^{2}\left(E_{L}\right)\right), \\
v_{m} & \rightarrow v \quad \text { weak }^{*} \text { in } L^{\infty}\left(0, T ; J_{\sigma}^{1}\left(E_{L}\right)\right), \\
\frac{\partial v_{m}}{\partial t} & \rightarrow \frac{\partial v}{\partial t} \quad \text { weakly in } L^{2}\left(0, T ; J_{\sigma}^{0}\left(E_{L}\right)\right),
\end{aligned}
$$


for some function $v(x, t)$, which satisfies (2-8) to (2-10). We note that $v \in$ $C\left([0, T] ; J_{\sigma}^{1}\left(E_{L}\right)\right)$, possibly after a modification on a set of measure zero in $[0, T]$. By repeatedly extracting a subsequence if necessary, we may assume that $\varepsilon_{i j}\left(v_{m}\right)$ converges to $\varepsilon_{i j}(v)$ for almost all $(x, t) \in E_{L} \times[0, T]$, for each $i, j=1,2$, since $v_{m} \rightarrow v$ strongly in $L^{2}\left(0, T ; J_{\sigma}^{1}\left(E_{L}\right)\right)$, which follows from (2-38), (2-40) and Aubin's compactness Lemma (see Lions [5, p. 57]). With the help of Lemma 1.3 of Lions [5, p. 12], we are led to conclude that for each $i, j=1,2$.

$$
D\left(v_{m}\right)^{-1+\eta} \varepsilon_{i j}\left(v_{m}\right) \rightarrow D(v)^{-1+\eta} \varepsilon_{i j}(v) \text { weakly in } L^{2}\left(E_{L} \times[0, T]\right) .
$$

Since $v_{m} \rightarrow v$ strongly in $L^{2}\left(0, T ; J_{\sigma}^{1}\left(E_{L}\right)\right)$, we see that $v_{m}(x, t) \rightarrow v(x, t)$ strongly in $J_{\sigma}^{\mathbf{1}}\left(E_{L}\right)$ for almost all $t \in[0, T]$ by extracting a subsequence. Hence, by letting $m \rightarrow \infty,(2-33)$ yields

$$
\begin{aligned}
& \int_{0}^{t} \int_{E_{L}}\left|\frac{\partial v}{\partial t}\right|^{2} d x d t+\mu \int_{E_{L}}|\nabla v|^{2} d x+2 \nu j_{L, \eta}(v(x, t)) \\
& \leq M(\mu, K)+\nu M(K), \quad \text { for almost all } t \in[0, T]
\end{aligned}
$$

where $M(\mu, K)$ and $M(K)$ are positive constants independent of $\nu, \eta$ and $L$. This shows (2-11).

Now we shall prove that $v(x, 0)=v_{0}(x)$. For each $k$ and $m$,

$$
\left\langle v_{m}(x, t), \varphi_{k}(x)\right\rangle=\left\langle v_{m}(x, 0), \varphi_{k}(x)\right\rangle+\int_{0}^{t}\left\langle\frac{\partial v_{m}}{\partial t}(x, t), \varphi_{k}(x)\right\rangle d t
$$

holds, where $\langle$,$\rangle denotes the inner product in L^{2}\left(E_{L}\right)^{2}$. Since $v_{m}(x, t) \rightarrow v(x, t)$ strongly in $J_{\sigma}^{1}\left(E_{L}\right)$ for almost all $t \in[0, T]$, and $\partial v_{m} / \partial t \rightarrow \partial v / \partial t$ weakly in $L^{2}\left(0, T ; J_{\sigma}^{0}\left(E_{L}\right)\right)$, it follows by letting $m \rightarrow \infty$ that

$$
\left\langle v(x, t), \varphi_{k}(x)\right\rangle=\left\langle v_{0}(x), \varphi_{k}(x)\right\rangle+\int_{0}^{t}\left\langle\frac{\partial v}{\partial t}(x, t), \varphi_{k}(x)\right\rangle d t
$$

holds for almost all $t \in[0, T]$. But $v(x, t) \in C\left([0, T] ; J_{\sigma}^{1}\left(E_{L}\right)\right)$ and hence,

$$
\left\langle v(x, 0), \varphi_{k}(x)\right\rangle=\left\langle v_{0}(x), \varphi_{k}(x)\right\rangle
$$

holds for all $k$, which implies $v(x, 0)=v_{0}(x)$. Finally we have to show that $(2-7)$ holds. On account of $(2-38)$ to $(2-41)$, it is apparent that

$$
\begin{aligned}
\int_{0}^{T}\langle & \left.\frac{\partial v}{\partial t}, \alpha(t) \varphi_{k}(x)\right\rangle d t+\int_{0}^{T}\left\langle(v \cdot \nabla) v, \alpha(t) \varphi_{k}(x)\right\rangle d t \\
& -\mu \int_{0}^{T}\left\langle\Delta v, \alpha(t) \varphi_{k}(x)\right\rangle d t+\nu \int_{0}^{T}\left(j_{L, \eta}^{\prime}(v), \alpha(t) \varphi_{k}(x)\right) d t \\
= & \int_{0}^{T}\left\langle F_{L}, \alpha(t) \varphi_{k}(x)\right\rangle d t
\end{aligned}
$$

holds for each $k$ and $\alpha(t) \in L^{2}(0, T)$, where $\langle$,$\rangle stands for the product in$ $L^{2}\left(E_{L}\right)^{2}$. For given $h(x, t) \in L^{2}\left(0, T ; J_{\sigma}^{1}\left(E_{L}\right)\right), \sum_{k=1}^{m} \alpha_{k}(t) \varphi_{k}(x) \rightarrow h(x, t)$ in 
$L^{2}\left(0, T ; J_{\sigma}^{1}\left(E_{L}\right)\right)$ as $m \rightarrow \infty$, where $\alpha_{k}(t)=\left\langle h(x, t), \varphi_{k}(x)\right\rangle$. Thus, for any $h(x, t) \in$ $L^{2}\left(0, T ; J_{\sigma}^{1}\left(E_{L}\right)\right)$, it holds that

$$
\begin{aligned}
\int_{0}^{T}\left\langle\frac{\partial v}{\partial t}, h(x, t)\right\rangle d t+\int_{0}^{T}\langle(v \cdot \nabla) v, h(x, t)\rangle d t \\
\quad-\mu \int_{0}^{T}\langle\Delta v, h(x, t)\rangle d t+\nu \int_{0}^{T}\left(j_{L, \eta}^{\prime}(v), h(x, t)\right) d t \\
=\int_{0}^{T}\left\langle F_{L}, h(x, t)\right\rangle d t .
\end{aligned}
$$

Now suppose $g(x, t) \in L^{2}\left(0, T ; H_{\sigma}^{1}\left(E_{L}\right)\right)$. Then, it is obvious that

$$
\tilde{g}(x, t)=g(x, t)-\frac{1}{L^{2}} \int_{E_{L}} g(x, t) d x
$$

belongs to $L^{2}\left(0, T ; J_{\sigma}^{1}\left(E_{L}\right)\right)$. Noting that

$$
\begin{gathered}
\int_{0}^{T}\langle\partial v / \partial t, c(t)\rangle d t=0, \quad \int_{0}^{T}\langle\Delta v, c(t)\rangle d t=0 \\
\int_{0}^{T}\langle(v \cdot \nabla) v, c(t)\rangle d t=0 \quad \text { and } \quad \int_{0}^{T}\left\langle F_{L}, c(t)\right\rangle d t=0
\end{gathered}
$$

hold for any $c(t) \in L^{2}(0, T)^{2}$, where $\langle$,$\rangle denotes the inner product in L^{2}\left(E_{L}\right)^{2}$ and recalling (2-13), we conclude that (2-7) holds for every $g \in L^{2}\left(0, T ; H_{\sigma}^{1}\left(E_{L}\right)\right)$.

2.2. Passing $L \rightarrow \infty$. In this subsection we still fix $0<\eta<1$ and shall expand $E_{L}$ to the whole space $R^{2}$. Through this process, the solutions obtained in Proposition 2.3 converge to a function in the appropriate sense. We state

PROPOSITION 2.5. Let $u_{0}(x)$ and $F(x, t)$ be the initial datum and the external force, respectively, given in Theorem 2.2. There is a function $v(x, t)$ such that

(i) $v(x, t) \in C\left([0, T] ; \mathcal{H}_{\sigma}^{1}\left(R^{2}\right)\right) \cap L^{2}\left(0, T ; ﹎{\sigma}^{2}\left(R^{2}\right)\right),(\partial v / \partial t)(x, t) \in L^{2}\left(0, T ; ﹎{\sigma}^{0}\left(R^{2}\right)\right)$, $\varepsilon_{i j}(v) \in L^{\infty}\left(0, T ; L^{1+\eta}\left(R^{2}\right)\right)$, for $i, j=1,2$, and $v(x, 0)=u_{0}(x)$;

(ii) it holds that

$$
\begin{gathered}
\int_{0}^{T}\left\langle\frac{\partial v}{\partial t}, g\right\rangle d t+\int_{0}^{T}\langle(v \cdot \nabla) v, g\rangle d t+\mu \int_{0}^{T} a(v, g) d t \\
\quad+\nu \int_{0}^{T} j_{\eta}(v+g) d t-\nu \int_{0}^{T} j_{\eta}(v) d t \\
\geq \int_{0}^{T}\langle F, g\rangle d t
\end{gathered}
$$

for all $g \in L^{2}\left(0, T ; \mathcal{G}_{1+\eta}\right)$, where $\langle$,$\rangle denotes the inner product in L^{2}\left(R^{2}\right)^{2}$;

(iii) it holds that

$$
\begin{gathered}
\|v\|_{C\left([0, T] ; \mathcal{H}_{\sigma}^{1}\left(R^{2}\right)\right)} \leq M(\mu, K), \\
\|v\|_{L^{2}\left(0, T ; \mathcal{H}_{\sigma}^{2}\left(R^{2}\right)\right)} \leq M(\mu, K), \\
\|\partial v / \partial t\|_{L^{2}\left(0, T ; \mathcal{H}_{\sigma}^{\circ}\left(R^{2}\right)\right)} \leq M(\mu, K)+\nu M(K) \\
\nu j_{\eta}(v) \leq M(\mu, K)+\nu M(K), \quad \text { for almost all } t \in[0, T],
\end{gathered}
$$

where $M(\mu, K)$ and $M(K)$ stand for positive constants independent of $\nu$ and $\eta$; and the constant $K$ depends only on $u_{0}(x)$ and $F(x, t)$. 
ProOF. We shall use the notation $\chi(G)$ for the characteristic function of a set $G$. Let $\left\{v_{L, 0}(x)\right\}$ and $\left\{F_{L}(x, t)\right\}$ be sequences of functions such that $v_{L, 0}(x) \in J_{\sigma}^{2}\left(E_{L}\right)$, $F_{L}(x, t) \in C\left([0, T] ; J^{0}\left(E_{L}\right)\right)$ and

$$
\begin{gathered}
\chi\left(E_{L / 2}\right) v_{L, 0}(x) \rightarrow u_{0}(x) \quad \text { strongly in } L^{2}\left(R^{2}\right)^{2} \text { as } L \rightarrow \infty \\
\chi\left(E_{L / 2}\right) F_{L}(x, t) \rightarrow F(x, t) \text { strongly in } L^{2}\left(0, T ; L^{2}\left(R^{2}\right)^{2}\right) \text { as } L \rightarrow \infty \\
\left\|v_{L, 0}\right\|_{J_{\sigma}^{1}\left(E_{L}\right)}+\left\|D\left(v_{L, 0}\right)\right\|_{L^{1}\left(E_{L}\right)} \leq K \\
\left\|F_{L}\right\|_{L^{2}\left(0, T ; J^{0}\left(E_{L}\right)\right)} \leq K
\end{gathered}
$$

for some positive constant $K$ independent of $\eta$ and $L$. We shall construct the above sequences in $\S 2.4$. Let us denote by $v_{L}(x, t)$ the solution obtained in Proposition 2.3 with the initial datum $v_{L, 0}(x)$ and the external force $F_{L}(x, t)$ as above. By means of (2-9) and (2-10), we can extract a subsequence still denoted by $\left\{v_{L}\right\}$ such that for some function $v(x, t)$, as $L \rightarrow \infty$,

$$
\begin{gathered}
\chi\left(E_{L}\right) v_{L} \rightarrow v \quad \text { weakly in } L^{2}\left(0, T ; L^{2}\left(R^{2}\right)^{2}\right), \\
\chi\left(E_{L}\right) \Delta v_{L} \rightarrow \Delta v \quad \text { weakly in } L^{2}\left(0, T ; L^{2}\left(R^{2}\right)^{2}\right), \\
\chi\left(E_{L}\right) \frac{\partial v_{L}}{\partial t} \rightarrow \frac{\partial v}{\partial t} \quad \text { weakly in } L^{2}\left(0, T ; L^{2}\left(R^{2}\right)^{2}\right) .
\end{gathered}
$$

It follows that $v \in C\left([0, T] ; W^{1,2}\left(R^{2}\right)^{2}\right)$ possibly after a modification on a set of measure zero in $[0, T]$. Let us choose any $\alpha(t) \in L^{2}(0, T)$ and $\psi(x) \in C_{0}^{\infty}\left(R^{2}\right)$. Noticing that $\operatorname{supp} \psi \subset E_{L}$ for large $L$, we have

$$
\begin{aligned}
& \int_{0}^{T}\langle v, \alpha(t) \nabla \psi(x)\rangle d t=\lim _{L \rightarrow \infty} \int_{0}^{T}\left\langle\chi\left(E_{L}\right) v_{L}, \alpha(t) \nabla \psi(x)\right\rangle d t \\
& \quad=\lim _{L \rightarrow \infty} \int_{0}^{T}\left\langle v_{L}, \alpha(t) \nabla \psi(x)\right\rangle d t=0, \quad \text { since } v_{L} \in C\left([0, T] ; J_{\sigma}^{1}\left(E_{L}\right)\right) .
\end{aligned}
$$

Here, the first two brackets denote the inner product in $L^{2}\left(R^{2}\right)^{2}$ and the third one stands for the inner product in $L^{2}\left(E_{L}\right)^{2}$. From (2-60) and the fact that $v \in$ $C\left([0, T] ; W^{1,2}\left(R^{2}\right)^{2}\right)$, we deduce that $\nabla \cdot v=0$ for all $t \in[0, T]$, and consequently, $v \in L^{2}\left(0, T ; \not \not_{\sigma}^{2}\left(R^{2}\right)\right) \cap C\left([0, T] ; \not \mathscr{H}_{\sigma}^{1}\left(R^{2}\right)\right)$ and $\partial v / \partial t \in L^{2}\left(0, T ; \not \nvdash_{\sigma}^{0}\left(R^{2}\right)\right)$. Now $(2-49),(2-50)$ and (2-51) follow from (2-8), (2-9) and (2-10). In the meantime, it is clear that $\chi\left(E_{L}\right) \varepsilon_{i j}\left(v_{L}\right) \rightarrow \varepsilon_{i j}(v)$ in $D^{\prime}\left((0, T) \times R^{2}\right)$, for each $i, j=1,2$. But (2-11) holds for each $v_{L}$ and thus, we obtain by applying the Banach-Alaoglu theorem

$$
\nu \int_{R^{2}} D(v)^{1+\eta} d x \leq M(\mu, K)+\nu M(K), \quad \text { for almost all } t \in[0, T],
$$

where $M(\mu, K)$ and $M(K)$ are the same as in (2-11). Next we shall prove that $v(x, 0)=u_{0}(x)$. Let $a(t) \in C^{1}([0, T])$ with $a(T)=0$ and $\psi(x) \in C_{0}^{\infty}\left(R^{2}\right)^{2}$. Then for each $L$ such that supp $\psi \subset$ interior of $E_{L / 2}$, it holds that

$$
\begin{array}{r}
\int_{0}^{T}\left\langle\chi\left(E_{L}\right) v_{L}, \frac{\partial}{\partial t} a(t) \psi(x)\right\rangle d t=\int_{0}^{T}\left\langle\chi\left(E_{L / 2}\right) v_{L}, \frac{\partial}{\partial t} a(t) \psi(x)\right\rangle d t \\
=\left\langle\chi\left(E_{L / 2}\right) v_{L, 0}, a(0) \psi(x)\right\rangle-\int_{0}^{T}\left\langle\chi\left(E_{L}\right) \frac{\partial v_{L}}{\partial t}, a(t) \psi(x)\right\rangle d t
\end{array}
$$


where $\langle$,$\rangle denotes the inner product in L^{2}\left(R^{2}\right)^{2}$. As $L \rightarrow \infty$, we observe that

$$
\int_{0}^{T}\left\langle v, \frac{\partial}{\partial t} a(t) \psi(x)\right\rangle d t=\left\langle u_{0}, a(0) \psi(x)\right\rangle-\int_{0}^{T}\left\langle\frac{\partial v}{\partial t}, a(t) \psi(x)\right\rangle d t
$$

holds, which, together with the fact that $v \in C\left([0, T] ; \not_{\sigma}^{1}\left(R^{2}\right)\right)$, implies that $v(x, 0)$ $=u_{0}(x)$. It remains to prove the property (ii). Let $g(x, t)=\sum_{k=1}^{m} \alpha_{k}(t) \psi_{k}(x)$, where $\psi_{k}(x) \in S, \alpha_{k}(t) \in L^{2}(0, T), k=1, \ldots, m$, and supp $\psi_{k} \subset \Omega, k=1, \ldots, m$, for some bounded open subset $\Omega$ of $R^{2}$. For every large $L$ so that $\Omega \subset$ interior of $E_{L}$, we see from (2-7) that

$$
\begin{aligned}
\int_{0}^{T}\langle\chi & \left.\left(E_{L}\right) \frac{\partial v_{L}}{\partial t}, g\right\rangle d t+\int_{0}^{T}\left\langle\chi\left(E_{L}\right)\left(v_{L} \cdot \nabla\right) v_{L}, g\right\rangle d t-\mu \int_{0}^{T}\left\langle\chi\left(E_{L}\right) \Delta v_{L}, g\right\rangle d t \\
& \quad+\nu \int_{0}^{T} j_{L, \eta}\left(v_{L}+g\right) d t-\nu \int_{0}^{T} j_{L, \eta}\left(v_{L}\right) d t \\
& \geq \int_{0}^{T}\left\langle\chi\left(E_{L}\right) F_{L}, g\right\rangle d t,
\end{aligned}
$$

where $\langle$,$\rangle is the inner product in L^{2}\left(R^{2}\right)^{2}$. Let $\left\{\Omega_{n}\right\}_{n=1}^{\infty}$ be a sequence of bounded open subsets of $R^{2}$ such that $\Omega_{n} \subset \Omega_{n+1}$ and $\bigcup_{n=1}^{\infty} \Omega_{n}=R^{2}$. With the help of Aubin's compactness lemma, we may extract a subsequence still denoted by $\left\{v_{L}\right\}$ such that

$$
\int_{0}^{T} \int_{\Omega_{1}}\left|\nabla v_{L}-\nabla v\right|^{2} d x d t \rightarrow 0 \quad \text { as } L \rightarrow \infty .
$$

By further extracting a subsequence from the above one,

$$
\int_{0}^{T} \int_{\Omega_{2}}\left|\nabla v_{L}-\nabla v\right|^{2} d x d t \rightarrow 0 \quad \text { as } L \rightarrow \infty .
$$

By repeating this process for all subsequent $\Omega_{n}$ and by the standard diagonal process, we conclude that for each bounded measurable subset $G$ of $R^{2}$,

$$
\int_{0}^{T} \int_{G}\left|\nabla v_{L}-\nabla v\right|^{2} d x d t \rightarrow 0 \quad \text { as } L \rightarrow \infty .
$$

Now it is easily seen that as $L \rightarrow \infty$,

$$
\begin{aligned}
\int_{0}^{T} & \int_{E_{L}} D\left(v_{L}+g\right)^{1+\eta} d x d t-\int_{0}^{T} \int_{E_{L}} D\left(v_{L}\right)^{1+\eta} d x d t \\
\quad= & \int_{0}^{T} \int_{\Omega} D\left(v_{L}+g\right)^{1+\eta} d x d t-\int_{0}^{T} \int_{\Omega} D\left(v_{L}\right)^{1+\eta} d x d t
\end{aligned}
$$

converges to

$$
\begin{aligned}
\int_{0}^{T} & \int_{\Omega} D(v+g)^{1+\eta} d x d t-\int_{0}^{T} \int_{\Omega} D(v)^{1+\eta} d x d t \\
& =\int_{0}^{T} \int_{R^{2}} D(v+g)^{1+\eta} d x d t-\int_{0}^{T} \int_{R^{2}} D(v)^{1+\eta} d x d t
\end{aligned}
$$


where we recall that $\operatorname{supp} \psi_{k}(x) \subset \Omega$ for $k=1, \ldots, m$. At the same time, by making use of (2-67) and the fact that $\chi\left(E_{L}\right) v_{L} \rightarrow v$ weak $^{*}$ in $L^{\infty}\left(0, T ; L^{2}\left(R^{2}\right)^{2}\right)$, we find that

$$
\int_{0}^{T}\left\langle\chi\left(E_{L}\right)\left(v_{L} \cdot \nabla\right) v_{L}, g\right\rangle_{L^{2}\left(R^{2}\right)^{2}} d t=\int_{0}^{T}\left\langle\left(v_{L} \cdot \nabla\right) v_{L}, g\right\rangle_{L^{2}(\Omega)^{2}} d t
$$

converges to

$$
\int_{0}^{T}\langle(v \cdot \nabla) v, g\rangle_{L^{2}(\Omega)^{2}} d t=\int_{0}^{T}\langle(v \cdot \nabla), g\rangle_{L^{2}\left(R^{2}\right)^{2}} d t
$$

as $L \rightarrow \infty$. Therefore, by letting $L \rightarrow \infty$, we deduce that

$$
\begin{gathered}
\int_{0}^{T}\left\langle\frac{\partial v}{\partial t}, g\right\rangle d t+\int_{0}^{T}\langle(v \cdot \nabla) v, g\rangle d t-\mu \int_{0}^{T}\langle\Delta v, g\rangle d t \\
\quad+\nu \int_{0}^{T} j_{\eta}(v+g) d t-\nu \int_{0}^{T} j_{\eta}(v) d t \\
\geq \int_{0}^{T}\langle F, g\rangle d t
\end{gathered}
$$

where $\langle$,$\rangle denotes the inner product in L^{2}\left(R^{2}\right)^{2}$. Since $S$ is dense in $\mathcal{G}_{1+\eta}$ for each $\eta>0$ by Lemma 1.7 and 1.9 , it is obvious that for each $g \in L^{2}\left(0, T ; \mathcal{G}_{1+\eta}\right)$, there is a sequence $\left\{g_{m}\right\}_{m=1}^{\infty}$ such that $g_{m}=\sum_{k=1}^{m} \beta_{m k}(t) \psi_{k}(x), \beta_{m k}(t) \in L^{2}(0, T)$, $\psi_{k}(x) \in \mathcal{S}, k=1, \ldots, m$, and $g_{m} \rightarrow g$ strongly in $L^{2}\left(0, T ; \mathcal{G}_{1+\eta}\right)$. (2-72) is valid for each $g_{m}$ as shown above and consequently, it is still true for all $g \in L^{2}\left(0, T ; \mathcal{G}_{1+\eta}\right)$. Now (2-48) follows from (2-72) and the identity $a(v, g)=-\langle\Delta v, g\rangle$.

2.3. Passing $\eta \rightarrow 0$. We shall let $\eta$ tend to zero and establish

PROPOSITION 2.6. Let $u_{0}(x)$ and $F(x, t)$ be the same as in Theorem 2.2. There is a function $u(x, t)$ such that

(i) $u(x, t) \in C\left([0, T] ; ﹎{\sigma}^{1}\left(R^{2}\right)\right) \cap L^{2}\left(0, T ; ﹎{\sigma}^{2}\left(R^{2}\right)\right), \partial u(x, t) / \partial t \in L^{2}\left(0, T ; ﹎{\sigma}^{0}\left(R^{2}\right)\right)$, $\varepsilon_{i j}(u) \in L^{\infty}\left(0, T ; L^{1}\left(R^{2}\right)\right), i, j=1,2$, and $u(x, 0)=u_{0}(x)$;

(ii) it holds that

$$
\begin{aligned}
& \int_{0}^{T}\left\langle\frac{\partial u}{\partial t}, g\right\rangle d t+\int_{0}^{T}\langle(u \cdot \nabla) u, g\rangle d t \\
& \quad+\mu \int_{0}^{T} a(u, g) d t+\nu \int_{0}^{T} j(u+g) d t-\nu \int_{0}^{T} j(u) d t \\
& \quad \geq \int_{0}^{T}\langle F, g\rangle d t,
\end{aligned}
$$

for all $g \in L^{2}\left(0, T ; \mathcal{G}_{1}\right)$, where $\langle$,$\rangle is the inner product in L^{2}\left(R^{2}\right)^{2}$;

(iii) it holds that

$$
\begin{gathered}
\|u\|_{C\left([0, T] ; \psi_{\sigma}^{1}\left(R^{2}\right)\right)} \leq M(\mu, K), \\
\|u\|_{L^{2}\left(0, T ; \aleph_{\sigma}^{2}\left(R^{2}\right)\right)} \leq M(\mu, K) \\
\|\partial u / \partial t\|_{L^{2}\left(0, T ; \nvdash_{\sigma}^{\circ}\left(R^{2}\right)\right)} \leq M(\mu, K)+\nu M(K), \\
\nu \int_{R^{2}} D(u) d x \leq M(\mu, K)+\nu M(K), \text { for almost all } t \in[0, T],
\end{gathered}
$$


where the constant $K$ depends only on $u_{0}(x), F(x, t)$; and $M(\mu, K), M(K)$ are positive constants independent of $\nu$.

PrOOF. Let us denote by $v_{\eta}$ the solution obtained in Proposition 2.5 for each $0<\eta<1$. By $(2-49)$ to $(2-51)$, we can extract a subsequence still denoted by $\left\{v_{\eta}\right\}$ such that as $\eta \rightarrow 0$,

$$
\begin{aligned}
v_{\eta} & \rightarrow u \quad \text { weakly in } L^{2}\left(0, T ; \nvdash_{\sigma}^{2}\left(R^{2}\right)\right), \\
v_{\eta} & \rightarrow u \quad \text { weak }^{*} \text { in } L^{\infty}\left(0, T ; \mathscr{H}_{\sigma}^{1}\left(R^{2}\right)\right), \\
\frac{\partial v_{\eta}}{\partial t} & \rightarrow \frac{\partial u}{\partial t} \quad \text { weakly in } L^{2}\left(0, T ; \not_{\sigma}^{0}\left(R^{2}\right)\right),
\end{aligned}
$$

for some function $u(x, t)$. It follows that $u(x, t) \in C\left([0, T] ; \not_{\sigma}^{1}\left(R^{2}\right)\right)$ possibly after a modification on a set of measure zero in $[0, T]$. As in the above section, it can be easily shown that $u(x, 0)=u_{0}(x)$. Now (2-74), (2-75) and (2-76) follow from $(2-49),(2-50)$ and (2-51). We may further extract by Aubin's compactness lemma and the diagonal process a subsequence such that

$$
v_{\eta} \rightarrow u \text { strongly in } L^{2}\left(0, T ; W^{1,2}(\Omega)^{2}\right)
$$

for each bounded open subset $\Omega$ of $R^{2}$. Let us choose any bounded open subset $\Omega$ of $R^{2}$. Then, there is a subsequence extracted from the above subsequence such that for almost all $t \in[0, T], v_{\eta}(x, t) \rightarrow u(x, t)$ strongly in $W^{1,2}(\Omega)^{2}$. Consequently, we have

$$
\begin{aligned}
\nu \int_{\Omega} D(u) d x & =\lim _{\eta \rightarrow 0} \nu \int_{\Omega} D\left(v_{\eta}\right) d x \\
& \leq \lim _{\eta \rightarrow 0} \nu\left(\int_{\Omega} D\left(v_{\eta}\right)^{1+\eta} d x\right)^{1 /(1+\eta)} \quad(\text { measure of } \Omega)^{\eta /(1+\eta)},
\end{aligned}
$$

using (2-52),

$$
\leq M(\mu, K)+\nu M(K)
$$

holds for almost all $t \in[0, T]$, where $M(\mu, K)$ and $M(K)$ are positive constants independent of $\nu$ and $\Omega$. Thus we infer

$$
\nu \int_{R^{2}} D(u) d x \leq M(\mu, K)+\nu M(K), \quad \text { for almost all } t \in[0, T],
$$

which proves (2-77). Next we shall establish (2-73). Choose any $g \in L^{2}\left(0, T ; \mathcal{G}_{1}\right)$. We first notice by Hölder's inequality

$$
\begin{aligned}
\left\|\varepsilon_{i j}(g)\right\|_{L^{2}\left(0, T ; L^{1+\eta}\left(R^{2}\right)\right)}^{2} \leq \int_{0}^{T}\left(\int_{R^{2}} D(g)^{1+\eta} d x\right)^{2 /(1+\eta)} d t \\
\quad \leq \int_{0}^{T}\left(\int_{R^{2}} D(g) d x\right)^{(2-2 \eta) /(1+\eta)}\left(\int_{R^{2}} D(g)^{2} d x\right)^{2 \eta /(1+\eta)} d t \\
\quad \leq\left(\int_{0}^{T}\left(\int_{R^{2}} D(g) d x\right)^{2} d t\right)^{(1-\eta) /(1+\eta)}\left(\int_{0}^{T} \int_{R^{2}} D(g)^{2} d x d t\right)^{2 \eta /(1+\eta)} \\
\leq M(g), \quad i, j=1,2,
\end{aligned}
$$

where $M(g)$ is a positive constant independent of $0 \leq \eta<1$. Using $\psi_{r}(x)$ defined 
by (1-35), we put $g_{r}(x, t)=g(x, t) \psi_{r}(x)$. Then, it follows from (1-36) and its analog for $\varepsilon_{i j}\left(g_{r}\right)$ that for almost all $t \in[0, T]$,

$$
\begin{aligned}
& \left\|g_{r}(x, t)-g(x, t)\right\|_{W^{1,2}\left(R^{2}\right)^{2}} \rightarrow 0 \text { as } r \rightarrow \infty \\
& \left\|\varepsilon_{i j}\left(g_{r}\right)-\varepsilon_{i j}(g)\right\|_{L^{1}\left(R^{2}\right)} \rightarrow 0 \text { as } r \rightarrow \infty, i, j=1,2, \\
& \left\|g_{r}(x, t)\right\|_{W^{1,2}\left(R^{2}\right)^{2}} \leq C\|g(x, t)\|_{W^{1,2}\left(R^{2}\right)^{2}}, \text { for all } r \geq 1, \\
& \left\|\varepsilon_{i j}\left(g_{r}\right)\right\|_{L^{1}\left(R^{2}\right)} \leq C\left(\left\|\varepsilon_{i j}(g)\right\|_{L^{1}\left(R^{2}\right)}+\|g\|_{L^{2}\left(R^{2}\right)^{2}}\right) \\
& \qquad i, j=1,2, \text { for all } r \geq 1,
\end{aligned}
$$

where $C$ is a positive constant independent of $g, r$ and $t$. By the dominated convergence theorem, we see that as $r \rightarrow \infty$,

$$
\int_{0}^{T}\left\|g_{r}(x, t)-g(x, t)\right\|_{W^{1,2}\left(R^{2}\right)^{2}}^{2} d t \rightarrow 0
$$

and

$$
\int_{0}^{T}\left\|\varepsilon_{i j}\left(g_{r}\right)-\varepsilon_{i j}(g)\right\|_{L^{1}\left(R^{2}\right)}^{2} d t \rightarrow 0, \quad i, j=1,2 .
$$

We also observe the inequality

$$
\begin{aligned}
& \frac{1}{1+\eta}\left|\int_{0}^{T} \int_{R^{2}} D\left(v_{\eta}+g\right)^{1+\eta} d x d t-\int_{0}^{T} \int_{R^{2}} D\left(v_{\eta}+g_{r}\right)^{1+\eta} d x d t\right| \\
& \leq \frac{1}{1+\eta} \int_{0}^{T} \int_{R^{2}}\left|D\left(v_{\eta}+g\right)^{1+\eta}-D\left(v_{\eta}+g_{r}\right)^{1+\eta}\right| d x d t \\
& \quad \leq \int_{0}^{T} \int_{R^{2}}\left(D\left(v_{\eta}+g\right)^{\eta}+D\left(v_{\eta}+g_{r}\right)^{\eta}\right) D\left(g-g_{r}\right) d x d t
\end{aligned}
$$

(where we have used the inequality $\left|\alpha^{1+\eta}-\beta^{1+\eta}\right| \leq(1+\eta)\left(\alpha^{\eta}+\beta^{\eta}\right)|\alpha-\beta|$, for all $\alpha \geq 0, \beta \geq 0$ )

$$
\begin{aligned}
\leq & \left(\int_{0}^{T} \int_{R^{2}} D\left(v_{\eta}+g\right)^{1+\eta} d x d t\right)^{\eta /(1+\eta)}\left(\int_{0}^{T} \int_{R^{2}} D\left(g-g_{r}\right)^{1+\eta} d x d t\right)^{1 /(1+\eta)} \\
& +\left(\int_{0}^{T} \int_{R^{2}} D\left(v_{\eta}+g_{r}\right)^{1+\eta} d x d t\right)^{\eta /(1+\eta)}\left(\int_{0}^{T} \int_{R^{2}} D\left(g-g_{r}\right)^{1+\eta} d x d t\right)^{1 /(1+\eta)}
\end{aligned}
$$

where Hölder's inequality has been used. Now Minkowski's inequality and Hölder's 
inequality yield the following estimates:

$$
\begin{gathered}
\left(\int_{0}^{T} \int_{R^{2}} D\left(v_{\eta}+g\right)^{1+\eta} d x d t\right)^{1 /(1+\eta)} \leq\left(\int_{0}^{T} \int_{R^{2}} D\left(v_{\eta}\right)^{1+\eta} d x d t\right)^{1 /(1+\eta)} \\
+\left(\int_{0}^{T} \int_{R^{2}} D(g)^{1+\eta} d x d t\right)^{1 /(1+\eta)} \leq\left(\int_{0}^{T} \int_{R^{2}} D\left(v_{\eta}\right)^{1+\eta} d x d t\right)^{1 /(1+\eta)} \\
+\left(\int_{0}^{T} \int_{R^{2}} D(g)^{2} d x d t\right)^{\eta /(1+\eta)}\left(\int_{0}^{T} \int_{R^{2}} D(g) d x d t\right)^{(1-\eta) /(1+\eta)}
\end{gathered}
$$

$$
\begin{gathered}
\left(\int_{0}^{T} \int_{R^{2}} D\left(v_{\eta}+g_{r}\right)^{1+\eta} d x d t\right)^{1 /(1+\eta)} \leq\left(\int_{0}^{T} \int_{R^{2}} D\left(v_{\eta}\right)^{1+\eta} d x d t\right)^{1 /(1+\eta)} \\
+\left(\int_{0}^{T} \int_{R^{2}} D\left(g_{r}\right)^{2} d x d t\right)^{\eta /(1+\eta)}\left(\int_{0}^{T} \int_{R^{2}} D\left(g_{r}\right) d x d t\right)^{(1-\eta) /(1+\eta)}
\end{gathered}
$$

$$
\begin{aligned}
& \left(\int_{0}^{T} \int_{R^{2}} D\left(g-g_{r}\right)^{1+\eta} d x d t\right)^{1 /(1+\eta)} \\
& \quad \leq\left(\int_{0}^{T} \int_{R^{2}} D\left(g-g_{r}\right) d x d t\right)^{(1-\eta) /(1+\eta)}\left(\int_{0}^{T} \int_{R^{2}} D\left(g-g_{r}\right)^{2} d x d t\right)^{\eta /(1+\eta)} .
\end{aligned}
$$

Combining (2-52), (2-77) and (2-89) to (2-94), we deduce that for any given $\delta>0$, there is $N(\delta)>0$, independent of $0<\eta<1$, so that for all $r>N(\delta)$ and $0<\eta<1$,

$$
\frac{\nu}{1+\eta}\left|\int_{0}^{T} \int_{R^{2}} D\left(v_{\eta}+g\right)^{1+\eta} d x d t-\int_{0}^{T} \int_{R^{2}} D\left(v_{\eta}+g_{r}\right)^{1+\eta} d x d t\right| \leq \delta
$$

and

$$
\nu\left|\int_{0}^{T} \int_{R^{2}} D(u+g) d x d t-\int_{0}^{T} \int_{R^{2}} D\left(u+g_{r}\right) d x d t\right| \leq \delta
$$

Now fix any $\delta>0$ and $r>N(\delta)$. Suppose that supp $g_{r}$ is contained in a bounded open subset $\Omega$ of $R^{2}$. Again by means of Minkowski's inequality and Hölder's 
inequality, we see

$$
\begin{aligned}
& \varlimsup_{\eta \rightarrow 0} \mid\left(\int_{0}^{T} \int_{\Omega} D\left(v_{\eta}+g_{r}\right)^{1+\eta} d x d t\right)^{1 /(1+\eta)} \\
& \quad-\left(\int_{0}^{T} \int_{\Omega} D\left(u+g_{r}\right)^{1+\eta} d x d t\right)^{1 /(1+\eta)} \mid \\
& \leq \varlimsup_{\eta \rightarrow 0}\left(\int_{0}^{T} \int_{\Omega} D\left(v_{\eta}-u\right)^{1+\eta} d x d t\right)^{1 /(1+\eta)} \mid \\
& \leq \varlimsup_{\eta \rightarrow 0}(\operatorname{measure} \Omega)^{(1-\eta) /(2+2 \eta)} T^{(1-\eta) /(2+2 \eta)}\left(\int_{0}^{T} \int_{\Omega} D\left(v_{\eta}-u\right)^{2} d x d t\right)^{1 / 2} \\
& \quad=0, \quad \text { by }(2-81) ;
\end{aligned}
$$

and, by the dominated convergence theorem,

$$
\lim _{\eta \rightarrow 0} \int_{0}^{T} \int_{\Omega} D\left(u+g_{r}\right)^{1+\eta} d x d t=\int_{0}^{T} \int_{\Omega} D\left(u+g_{r}\right) d x d t .
$$

Hence, we obtain

$$
\lim _{\eta \rightarrow 0} \int_{0}^{T} \int_{\Omega} D\left(v_{\eta}+g_{r}\right)^{1+\eta} d x d t=\int_{0}^{T} \int_{\Omega} D\left(u+g_{r}\right) d x d t
$$

and similarly,

$$
\lim _{\eta \rightarrow 0} \int_{0}^{T} \int_{\Omega} D\left(v_{\eta}\right)^{1+\eta} d x d t=\int_{0}^{T} \int_{\Omega} D(u) d x d t .
$$

It is now apparent that

$$
\begin{aligned}
\nu \int_{0}^{T} & \int_{R^{2}} D\left(u+g_{r}\right) d x d t-\nu \int_{0}^{T} \int_{R^{2}} D(u) d x d t \\
& =\nu \int_{0}^{T} \int_{\Omega} D\left(u+g_{r}\right) d x d t-\nu \int_{0}^{T} \int_{\Omega} D(u) d x d t \\
& =\lim _{\eta \rightarrow 0} \frac{\nu}{1+\eta}\left(\int_{0}^{T} \int_{\Omega} D\left(v_{\eta}+g_{r}\right)^{1+\eta} d x d t-\int_{0}^{T} \int_{\Omega} D\left(v_{\eta}\right)^{1+\eta} d x d t\right) \\
& =\lim _{\eta \rightarrow 0} \frac{\nu}{1+\eta}\left(\int_{0}^{T} \int_{R^{2}} D\left(v_{\eta}+g_{r}\right)^{1+\eta} d x d t-\int_{0}^{T} \int_{R^{2}} D\left(v_{\eta}\right)^{1+\eta} d x d t\right) \\
& \geq \varlimsup_{\eta \rightarrow 0} \frac{\nu}{1+\eta}\left(\int_{0}^{T} \int_{R^{2}} D\left(v_{\eta}+g\right)^{1+\eta} d x d t-\int_{0}^{T} \int_{R^{2}} D\left(v_{\eta}\right)^{1+\eta} d x d t\right)-\delta .
\end{aligned}
$$


Combining (2-96) and (2-101), we conclude (2-102)

$$
\begin{aligned}
& \nu \int_{0}^{T} \int_{R^{2}} D(u+g) d x d t-\nu \int_{0}^{T} \int_{R^{2}} D(u) d x d t \\
& \quad \geq \varlimsup_{\eta \rightarrow 0} \frac{\nu}{1+\eta}\left(\int_{0}^{T} \int_{R^{2}} D\left(v_{\eta}+g\right)^{1+\eta} d x d t-\int_{0}^{T} \int_{R^{2}} D\left(v_{\eta}\right)^{1+\eta} d x d t\right)-2 \delta,
\end{aligned}
$$

and, since $\delta$ was arbitrarily chosen,

$(2-103)$

$$
\begin{aligned}
& \nu \int_{0}^{T} \int_{R^{2}} D(u+g) d x d t-\nu \int_{0}^{T} \int_{R^{2}} D(u) d x d t \\
& \quad \geq \varlimsup_{\eta \rightarrow 0} \frac{\nu}{1+\eta}\left(\int_{0}^{T} \int_{R^{2}} D\left(v_{\eta}+g\right)^{1+\eta} d x d t-\int_{0}^{T} \int_{R^{2}} D\left(v_{\eta}\right)^{1+\eta} d x d t\right) .
\end{aligned}
$$

In the meantime, (2-79) and (2-81) yield

$$
\lim _{\eta \rightarrow 0} \int_{0}^{T}\left\langle\left(v_{\eta} \cdot \nabla\right) v_{\eta}, g_{r}\right\rangle d t=\int_{0}^{T}\left\langle(u \cdot \nabla) u, g_{r}\right\rangle d t
$$

for each $r>0$, where $\langle$,$\rangle is the inner product in L^{2}\left(R^{2}\right)^{2}$, which, together with (2-78), (2-79) and (2-89), implies

$$
\lim _{\eta \rightarrow 0} \int_{0}^{T}\left\langle\left(v_{\eta} \cdot \nabla\right) v_{\eta}, g\right\rangle d t=\int_{0}^{T}\langle(u \cdot \nabla) u, g\rangle d t .
$$

Since each $v_{\eta}$ satisfies (2-48) with this $g$, we combine (2-78), (2-80), (2-103) and (2-105) to arrive at

$$
\begin{aligned}
& \int_{0}^{T}\left\langle\frac{\partial u}{\partial t}, g\right\rangle d t+\int_{0}^{T}\langle(u \cdot \nabla) u, g\rangle d t \\
& \quad+\mu \int_{0}^{T} a(u, g) d t+\nu \int_{0}^{T} j(u+g) d t-\nu \int_{0}^{T} j(u) d t \\
& \quad \geq \int_{0}^{T}\langle F, g\rangle d t
\end{aligned}
$$

for all $g \in L^{2}\left(0, T ; \mathcal{G}_{1}\right)$, where $\langle$,$\rangle is the inner product in L^{2}\left(R^{2}\right)^{2}$.

2.4. Proof of Theorem 2.2. Let $u_{0}(x)$ be given in $\mathcal{G}_{1,0}$. Then, there is a sequence $\left\{w_{k}(x)\right\}$ in $S$ such that $w_{k}(x)$ converges to $u_{0}(x)$ in the $\mathcal{G}_{1}$ norm. We can construct a sequence of increasing positive number $\left\{L_{k}\right\}$ such that $\operatorname{supp} w_{k}(x)$ Cinterior of $E_{L_{k} / 2}$ for each $k$ and $L_{k} \rightarrow \infty$ as $k \rightarrow \infty$. Now we define $v_{L_{k}, 0}(x)$ as follows:

(i) for $-L_{k} / 2 \leq x_{1} \leq-L_{k} / 4,-L_{k} / 2 \leq x_{2} \leq-L_{k} / 4$,

$$
v_{L_{k}, 0}\left(x_{1}, x_{2}\right)=w_{k}\left(x_{1}+L_{k} / 2, x_{2}+L_{k} / 2\right)
$$

(ii) for $-L_{k} / 4 \leq x_{1} \leq L_{k} / 4,-L_{k} / 2 \leq x_{2} \leq-L_{k} / 4$,

$$
v_{L_{k}, 0}\left(x_{1}, x_{2}\right)=-w_{k}\left(x_{1}, x_{2}+L_{k} / 2\right) \text {; }
$$

(iii) for $L_{k} / 4 \leq x_{1} \leq L_{k} / 2,-L_{k} / 2 \leq x_{2} \leq-L_{k} / 4$,

$$
v_{L_{k}, 0}\left(x_{1}, x_{2}\right)=w_{k}\left(x_{1}-L_{k} / 2, x_{2}+L_{k} / 2\right) \text {; }
$$


(iv) for $-L_{k} / 2 \leq x_{1} \leq-L_{k} / 4,-L_{k} / 4 \leq x_{2} \leq L_{k} / 4$,

$$
v_{L_{k}, 0}\left(x_{1}, x_{2}\right)=-w_{k}\left(x_{1}+L_{k} / 2, x_{2}\right) ;
$$

(v) for $-L_{k} / 4 \leq x_{1} \leq L_{k} / 4,-L_{k} / 4 \leq x_{2} \leq L_{k} / 4$,

$$
v_{L_{k}, 0}\left(x_{1}, x_{2}\right)=w_{k}\left(x_{1}, x_{2}\right)
$$

(vi) for $L_{k} / 4 \leq x_{1} \leq L_{k} / 2,-L_{k} / 4 \leq x_{2} \leq L_{k} / 4$,

$$
v_{L_{k}, 0}\left(x_{1}, x_{2}\right)=-w_{k}\left(x_{1}-L_{k} / 2, x_{2}\right) ;
$$

(vii) for $-L_{k} / 2 \leq x_{1} \leq-L_{k} / 4, L_{k} / 4 \leq x_{2} \leq L_{k} / 2$,

$$
v_{L_{k}, 0}\left(x_{1}, x_{2}\right)=w_{k}\left(x_{1}+L_{k} / 2, x_{2}-L_{k} / 2\right) ;
$$

(viii) for $-L_{k} / 4 \leq x_{1} \leq L_{k} / 4, L_{k} / 4 \leq x_{2} \leq L_{k} / 2$,

$$
v_{L_{k}, 0}\left(x_{1}, x_{2}\right)=-w_{k}\left(x_{1}, x_{2}-L_{k} / 2\right)
$$

(ix) for $L_{k} / 4 \leq x_{1} \leq L_{k} / 2, L_{k} / 4 \leq x_{2} \leq L_{k} / 2$,

$$
v_{L_{k}, 0}\left(x_{1}, x_{2}\right)=w_{k}\left(x_{1}-L_{k} / 2, x_{2}-L_{k} / 2\right) \text {; }
$$

(x) for other $\left(x_{1}, x_{2}\right)$, define $v_{L_{k}, 0}\left(x_{1}, x_{2}\right)$ so that $v_{L_{k}, 0}\left(x_{1}, x_{2}\right)$ be $L_{k}$-periodic, i.e.,

$$
v_{L_{k}, 0}\left(x_{1}, x_{2}\right)=v_{L_{k}, 0}\left(x_{1}+L_{k}, x_{2}\right)=v_{L_{k}, 0}\left(x_{1}, x_{2}+L_{k}\right) .
$$

Then it is obvious that $v_{L_{k}, 0}(x) \in J_{\sigma}^{\infty}\left(E_{L_{k}}\right)$.

Furthermore, (2-53) holds and

$$
\left\|v_{L_{k}, 0}(x)\right\|_{J_{\sigma}^{1}\left(E_{L_{k}}\right)}+\left\|D\left(v_{L_{k}, 0}\right)\right\|_{L^{1}\left(E_{L_{k}}\right)} \leq K,
$$

where $K$ is a positive constant independent of $L_{k}$ and depends only on $\left\|u_{0}(x)\right\|_{\psi_{\sigma}^{1}\left(R^{2}\right)}$ and $\left\|D\left(u_{0}\right)\right\|_{L^{1}\left(R^{2}\right)}$.

Next we shall construct $F_{L_{k}}(x, t)$ for given $F(x, t)$ in $L^{2}\left(0, T ; L^{2}\left(R^{2}\right)^{2}\right)$. Since $C\left([0, T] ; L^{2}\left(R^{2}\right)^{2}\right)$ is dense in $L^{2}\left(0, T ; L^{2}\left(R^{2}\right)^{2}\right)$, we can find a sequence $\left\{G_{k}(x, t)\right\}$ in $C\left([0, T] ; L^{2}\left(R^{2}\right)^{2}\right)$ such that $G_{k}(x, t) \rightarrow F(x, t)$ strongly in $L^{2}\left(0, T ; L^{2}\left(R^{2}\right)^{2}\right)$. For each $t \in[0, T]$, we define $F_{L_{k}}(x, t)$ through the above procedure (i) to $(\mathrm{x})$ with $w_{k}$ replaced by $\chi\left(E_{L_{k} / 2}\right) G_{k}(x, t)$. Then it is easy to see that $F_{L_{k}}(x, t) \in$ $C\left([0, T] ; J^{0}\left(E_{L}\right)\right)$ and (2-54), (2-56) hold. With these data, we can use the results in $\S \S 2.1$ to 2.3. By the same argument as in Duvaut and Lions [1] , it can be shown that (2-73) implies (2-1); we omit the technical details. For the uniqueness of solution, we still borrow an argument from Duvaut and Lions [1]. Suppose $U_{1}(x, t)$ and $U_{2}(x, t)$ are strong solution of (0-1) to (0-3). By Lemma 1.10, $\mathcal{G}_{1}$ is separable and hence, there is a countable dense subset $\left\{w_{n}\right\}_{n=1}^{\infty}$ in $\mathcal{G}_{1}$. Let us write (2-1) for $U_{1}$ and each $w_{n}$ :

$$
\begin{aligned}
& \left\langle\frac{\partial U_{1}}{\partial t}, w_{n}-U_{1}\right\rangle+\left\langle\left(U_{1} \cdot \nabla\right) U_{1}, w_{n}-U_{1}\right\rangle+\mu a\left\langle U_{1}, w_{n}-U_{1}\right\rangle \\
& \quad+\nu \int_{R^{2}} D\left(w_{n}\right) d x-\nu \int_{R^{2}} D\left(U_{1}\right) d x \geq\left\langle F, w_{n}-U_{1}\right\rangle
\end{aligned}
$$


holds for every $t \in[0, T]-G_{n}$, where $G_{n}$ is a measurable subset of $[0, T]$ with measure $G_{n}=0$. Put $G=\bigcup_{n=1}^{\infty} G_{n}$. Then, measure $G=0$ and (2-108) holds for all $w_{n}$ at the same time for each $t \in[0, T]-G$, from which it follows that

$$
\begin{aligned}
& \left\langle\frac{\partial U_{1}}{\partial t}, U_{2}-U_{1}\right\rangle+\left\langle\left(U_{1} \cdot \nabla\right) U_{1}, U_{2}-U_{1}\right\rangle+\mu a\left(U_{1}, U_{2}-U_{1}\right) \\
& \quad+\nu j\left(U_{2}\right)-\nu j\left(U_{1}\right) \geq\left\langle F, U_{2}-U_{1}\right\rangle
\end{aligned}
$$

holds for almost all $t \in[0, T]$. In the same way,

$$
\begin{aligned}
& \left\langle\frac{\partial U_{2}}{\partial t}, U_{1}-U_{2}\right\rangle+\left\langle\left(U_{2} \cdot \nabla\right) U_{2}, U_{1}-U_{2}\right\rangle+\mu a\left(U_{2}, U_{1}-U_{2}\right) \\
& \quad+\nu j\left(U_{1}\right)-\nu j\left(U_{2}\right) \geq\left\langle F, U_{1}-U_{2}\right\rangle
\end{aligned}
$$

holds for almost all $t \in[0, T]$. Adding (2-109) and (2-110), we obtain

$$
\begin{aligned}
& \left\langle\frac{\partial U_{1}}{\partial t}-\frac{\partial U_{2}}{\partial t}, U_{1}-U_{2}\right\rangle+\left\langle\left(U_{1} \cdot \nabla\right) U_{1}-\left(U_{2} \cdot \nabla\right) U_{2}, U_{1}-U_{2}\right\rangle \\
& \quad+\mu a\left(U_{1}-U_{2}, U_{1}-U_{2}\right) \leq 0 .
\end{aligned}
$$

Using the inequality

$$
\begin{aligned}
\mid\left\langle\left(U_{1} \cdot \nabla\right) U_{1}-\right. & \left.\left(U_{2} \cdot \nabla\right) U_{2}, U_{1}-U_{2}\right\rangle \mid \\
& \leq\left|\left\langle\left(U_{1}-U_{2}\right) \cdot \nabla U_{1}, U_{1}-U_{2}\right\rangle\right|+\left|\left\langle U_{2} \cdot \nabla\left(U_{1}-U_{2}\right), U_{1}-U_{2}\right\rangle\right| \\
& =\left|\left\langle\left(U_{1}-U_{2}\right) \cdot \nabla U_{1}, U_{1}-U_{2}\right\rangle\right| \\
& \leq C\left\|\nabla U_{1}\right\|_{L^{2}\left(R^{2}\right)}\left\|U_{1}-U_{2}\right\|_{L^{4}\left(R^{2}\right)}^{2} \\
& \leq C\left\|\nabla U_{1}\right\|_{L^{2}\left(R^{2}\right)}\left\|U_{1}-U_{2}\right\|_{L^{2}\left(R^{2}\right)}\left\|\nabla\left(U_{1}-U_{2}\right)\right\|_{L^{2}\left(R^{2}\right)} \\
& \leq \frac{\mu}{2}\left\|\nabla\left(U_{1}-U_{2}\right)\right\|_{L^{2}\left(R^{2}\right)}^{2}+\frac{C^{2}}{2 \mu}\left\|\nabla U_{1}\right\|_{L^{2}\left(R^{2}\right)}^{2}\left\|U_{1}-U_{2}\right\|_{L^{2}\left(R^{2}\right)}^{2},
\end{aligned}
$$

where $C$ stands for positive constants independent of $U_{1}$ and $U_{2}$, we derive from $(2-111)$

$$
\begin{aligned}
\frac{d}{d t}\left\|U_{1}-U_{2}\right\|_{L^{2}\left(R^{2}\right)}^{2} & +\mu\left\|\nabla\left(U_{1}-U_{2}\right)\right\|_{L^{2}\left(R^{2}\right)}^{2} \\
& \leq \frac{C^{2}}{\mu}\left\|\nabla U_{1}\right\|_{L^{2}\left(R^{2}\right)}^{2}\left\|U_{1}-U_{2}\right\|_{L^{2}\left(R^{2}\right)}^{2} .
\end{aligned}
$$

Since $U_{1}$ is a strong solution, $\left\|\nabla U_{1}\right\|_{L^{2}\left(R^{2}\right)}^{2} \in L^{\infty}(0, T)$. Now the Gronwall inequality yields the uniqueness.

3. Convergence of solutions as $\nu \rightarrow 0$. As mentioned in the introduction, the inequality (0-1) reduces to the Navier-Stokes equations when the yield limit $\nu$ becomes zero. So we naturally expect the strong solution of $(0-1)$ to $(0-3)$ with $\nu>0$ to converge to the solution of the Navier-Stokes equation as $\nu$ tends to zero. Our assertion is

THEOREM 3.1. Let $u_{0}(x) \in \mathcal{G}_{1,0}$ and $F(x, t) \in L^{2}\left(0, T ; L^{2}\left(R^{2}\right)^{2}\right)$. Then the strong solution $u_{\nu}(x, t)$ of $(0-1)$ to (0-3) converges to the solution $u(x, t)$ of the 
Navier-Stokes equations as $\nu$ tends to zero. A more precise statement is, as $\nu \rightarrow 0$,

$$
\begin{aligned}
u_{\nu} & \rightarrow u \quad \text { weakly in } L^{2}\left(0, T ; \not_{\sigma}^{2}\left(R^{2}\right)\right), \\
u_{\nu} & \rightarrow u \quad \text { weak } k^{*} \text { in } L^{\infty}\left(0, T ; \mathscr{H}_{\sigma}^{1}\left(R^{2}\right)\right), \\
\frac{\partial u_{\nu}}{\partial t} & \rightarrow \frac{\partial u}{\partial t} \quad \text { weakly in } L^{2}\left(0, T ; \nvdash_{\sigma}^{0}\left(R^{2}\right)\right) .
\end{aligned}
$$

PROOF. Owing to the uniqueness of strong solution, the estimates (2-74) to $(2-77)$ are satisfied by $u_{\nu}(x, t)$, for each $\nu>0$. Hence, we can extract a subsequence still denoted by $\left\{u_{\nu}\right\}$ such that as $\nu \rightarrow 0$,

$$
\begin{aligned}
u_{\nu} & \rightarrow u \quad \text { weakly in } L^{2}\left(0, T ; \nvdash_{\sigma}^{2}\left(R^{2}\right)\right), \\
u_{\nu} & \rightarrow u \quad \text { weak }^{*} \text { in } L^{\infty}\left(0, T ; \not_{\sigma}^{1}\left(R^{2}\right)\right), \\
\frac{\partial u_{\nu}}{\partial t} & \rightarrow \frac{\partial u}{\partial t} \quad \text { weakly in } L^{2}\left(0, T ; \nvdash_{\sigma}^{0}\left(R^{2}\right)\right)
\end{aligned}
$$

for some function $u(x, t)$. It follows that $u \in C\left([0, T] ; \not \nvdash_{\sigma}^{1}\left(R^{2}\right)\right)$ possibly after a modification on a set of measure zero in $[0, T]$. By an analogous argument as in $\S 2.2$, we can easily show that $u(x, 0)=u_{0}(x)$. As before, we use Aubin's compactness lemma and the diagonal process to extract a further subsequence so that for each bounded open subset $\Omega$ of $R^{2}$,

$$
u_{\nu}(x, t) \rightarrow u(x, t) \quad \text { strongly in } L^{2}\left(0, T ; W^{1,2}(\Omega)^{2}\right) .
$$

By the same argument as in $\S 2.4$, we may put $w=u_{\nu}(x, t)+b(t) h(x)$ in $(2-1)$, where $h(x) \in S$ and $b(t) \in L^{2}(0, T)$, so that

$$
\begin{aligned}
\int_{0}^{T}\langle & \left.\frac{\partial u_{\nu}}{\partial t}, b(t) h(x)\right\rangle d t+\mu \int_{0}^{T} a\left(u_{\nu}, b(t) h(x)\right) d t \\
& \quad+\int_{0}^{T}\left\langle\left(u_{\nu} \cdot \nabla\right) u_{\nu}, b(t) h(x)\right\rangle d t+\nu \int_{0}^{T} j\left(u_{\nu}+b(t) h(x)\right) d t-\nu \int_{0}^{T} j\left(u_{\nu}\right) d t \\
& \geq \int_{0}^{T}\langle F, b(t) h(x)\rangle d t
\end{aligned}
$$

holds. On account of (3-7), we find that

$$
\begin{aligned}
\lim _{\nu \rightarrow 0} & \left(\int_{0}^{T} \int_{R^{2}} D\left(u_{\nu}+b(t) h(x)\right) d x d t-\int_{0}^{T} \int_{R^{2}} D\left(u_{\nu}\right) d x d t\right) \\
& =\lim _{\nu \rightarrow 0}\left(\int_{0}^{T} \int_{\Omega} D\left(u_{\nu}+b(t) h(x)\right) d x d t-\int_{0}^{T} \int_{\Omega} D\left(u_{\nu}\right) d x d t\right) \\
& =\int_{0}^{T} \int_{\Omega} D(u+b(t) h(x)) d x d t-\int_{0}^{T} \int_{\Omega} D(u) d x d t
\end{aligned}
$$

where supp $h \subset \Omega$, and

$$
\lim _{\nu \rightarrow 0} \int_{0}^{T}\left\langle\left(u_{\nu} \cdot \nabla\right) u_{\nu}, b(t) h(x)\right\rangle d t=\int_{0}^{T}\langle(u \cdot \nabla) u, b(t) h(x)\rangle d t .
$$


We may now conclude that for each $h(x) \in S$ and $b(t) \in L^{2}(0, T)$,

$$
\begin{aligned}
\int_{0}^{T}\left\langle\frac{\partial u}{\partial t}, b(t) h(x)\right\rangle d t+\mu \int_{0}^{T} a(u, b(t) h(x)) d t \\
\quad+\int_{0}^{T}\langle(u \cdot \nabla) u, b(t) h(x)\rangle d t=\int_{0}^{T}\langle F, b(t) h(x)\rangle d t,
\end{aligned}
$$

from which it follows that for each $w(x) \in \mathcal{H}_{\sigma}^{1}\left(R^{2}\right)$,

$$
\langle\partial u / \partial t, w\rangle-\mu\langle\Delta u, w\rangle+\langle(u \cdot \nabla) u, w\rangle=\langle F, w\rangle
$$

holds for almost all $t \in[0, T]$, since $S$ is dense in $\nvdash_{\sigma}^{1}\left(R^{2}\right)$. Hence, $u(x, t)$ is a solution of the Navier-Stokes equations, and it can be easily shown that $u(x, t)$ is the unique solution in the above function class, from which (3-1), (3-2) and (3-3) follow.

4. Remark on the regularity of solutions. We shall present an initial datum which lies in $S$, but which does not generate a solution as regular in time variable near $t=0$ as the solution of the Navier-Stokes equations with the same initial condition. Let us define a velocity vector $u_{0}(x)$ as follows

$$
u_{0}(x)=(-f(r) \sin \theta, f(r) \cos \theta)
$$

where $x_{1}=r \cos \theta, x_{2}=r \sin \theta$, and

$$
f(r) \in C_{0}^{\infty}((0, \infty)), \quad \operatorname{supp} f \subset(0,2), \quad f(r)=e^{r} \text { for } \frac{1}{2} \leq r \leq \frac{3}{2} .
$$

Then, it is obvious that $u_{0}(x) \in S$, and our assertion is

THEOREM 4.1. Let $F(x, t) \equiv 0$ in (0-1). For the initial datum $u_{0}(x)$ given by (4-1), there is no strong solution of (0-1) to (0-3) satisfying further regularity

$$
u(x, t) \in C\left([0, T] ; \not \nvdash_{\sigma}^{2}\left(R^{2}\right)\right) \cap C^{1}\left([0, T] ; \not \nvdash_{\sigma}^{0}\left(R^{2}\right)\right)
$$

for some $T>0$.

REMARK 4.2. It is known that the solution of the Navier-Stokes equations with the above initial condition belongs to $C\left([0, T] ; \nvdash_{\sigma}^{m}\left(R^{2}\right)\right) \cap C^{1}\left([0, T] ; \nvdash_{\sigma}^{m-2}\left(R^{2}\right)\right)$ for any $m \geq 3$, for some $T>0$; see Kato [4] for the result in $R^{3}$, which is also valid in $R^{2}$.

We need some elementary technical lemmas.

LEMMA 4.3. Let $a, b \in R^{n}, n \geq 2$. Then, it holds that

$$
\left|\frac{|a+\lambda b|-|a|}{\lambda}\right| \leq|b|, \quad \text { for all } \lambda>0
$$

and if $|a| \neq 0$, then

$$
\frac{|a+\lambda b|-|a|}{\lambda} \rightarrow \frac{\langle a, b\rangle}{|a|} \quad \text { as } \lambda \rightarrow 0+
$$

where $\langle$,$\rangle is the inner product in R^{n}$. that

$$
\frac{|a+\lambda b|-|a|}{\lambda}=\frac{2 \lambda\langle a, b\rangle+\lambda^{2}|b|^{2}}{\lambda(|a+\lambda b|+|a|)},
$$

from which (4-5) follows. 
LEMMA 4.4. Let $\psi=\left(\psi_{1}, \psi_{2}\right)$ be a function in $R^{2}$ such that $D(\psi) \in L^{1}\left(R^{2}\right)$ and $\operatorname{supp} \psi \subset\left\{x: \frac{1}{2}<|x|<\frac{3}{2}\right\}$. Then, we have

$$
\frac{1}{\lambda}\left(\int_{R^{2}} D\left(u_{0}+\lambda \psi\right) d x-\int_{R^{2}} D\left(u_{0}\right) d x\right) \rightarrow \int_{R^{2}} \sum_{i, j=1}^{2} \frac{2 \varepsilon_{i j}\left(u_{0}\right) \varepsilon_{i j}(\psi)}{D\left(u_{0}\right)} d x
$$

as $\lambda \rightarrow 0+$, where $u_{0}(x)$ is defined by (4-1).

PROOF. First we observe that

$$
\begin{gathered}
\varepsilon_{11}\left(u_{0}\right)=\sin \theta \cos \theta(-d f(r) / d r+f(r) / r), \\
\varepsilon_{12}\left(u_{0}\right)=\frac{1}{2}\left(\cos ^{2} \theta-\sin ^{2} \theta\right)(d f(r) / d r-f(r) / r), \\
\varepsilon_{22}\left(u_{0}\right)=\sin \theta \cos \theta(d f(r) / d r-f(r) / r)
\end{gathered}
$$

and hence $D\left(u_{0}\right) \neq 0$ for $r \neq 1, \frac{1}{2} \leq r \leq \frac{3}{2}$. By means of Lemma 4.3 and the dominated convergence theorem, we derive (4-7).

Now we proceed to

PROOF OF THEOREM 4.1. Assume that there is a strong solution $u(x, t)$ of $(0-1)$ to (0-3) satisfying (4-3) for some $T>0$. Let us take $w=u(x, t)+h(x)$ in $(2-1)$, where $h(x) \in \mathcal{G}_{1}$ and $\operatorname{supp} h(x)$ is contained in a bounded open subset $\Omega$ of $R^{2}$, so that

$$
\left\langle\frac{\partial u}{\partial t}, h\right\rangle+\langle(u \cdot \nabla) u, h\rangle+\mu a(u, h)+\nu \int_{R^{2}} D(u+h) d x-\nu \int_{R^{2}} D(u) d x \geq 0
$$

holds for almost all $t \in[0, T]$. Since $u \in C\left([0, T] ; \nvdash_{\sigma}^{2}\left(R^{2}\right)\right) \cap C^{1}\left([0, T] ; \nvdash_{\sigma}^{0}\left(R^{2}\right)\right)$, it is evident that

$$
\begin{array}{rl}
\int_{R^{2}} & D(u(x, t)+h(x)) d x-\int_{R^{2}} D(u(x, t)) d x \\
= & \int_{\Omega} D(u(x, t)+h(x)) d x-\int_{\Omega} D(u(x, t)) d x
\end{array}
$$

is a continuous function of $f \in[0, T]$, and that

$$
\nu G(x, t) \stackrel{\text { def }}{=}-\partial u / \partial t-(u \cdot \nabla) u+\mu \Delta u
$$

belongs to $C\left([0, T] ; L^{2}\left(R^{2}\right)^{2}\right)$. Thus, it follows from (4-11) that

$$
\int_{R^{2}} D(u+h) d x-\int_{R^{2}} D(u) d x \geq\langle G, h\rangle
$$

holds for all $t \in[0, T]$, and, in particular,

$$
\int_{R^{2}} D\left(u_{0}(x)+h(x)\right) d x-\int_{R^{2}} D\left(u_{0}(x)\right) d x \geq\langle G(x, 0), h(x)\rangle
$$

holds for all $h(x) \in \mathcal{G}_{1}$ with compact support, where $G(x, 0)$ is a function of $L^{2}\left(R^{2}\right)^{2}$ and $\langle$,$\rangle is the inner product in L^{2}\left(R^{2}\right)^{2}$. Next we define $h_{n}(x), n \geq 4$, as follows:

$$
h_{n}(x)=\left(-g_{n}(r) \sin \theta, g_{n}(r) \cos \theta\right),
$$

where $x_{1}=r \cos \theta, x_{2}=r \sin \theta$ and

$$
g_{n}(r)= \begin{cases}n+1-n r & \text { for } 1 \leq r \leq 1+1 / n \\ 1-n+n r & \text { for } 1-1 / n \leq r \leq 1 \\ 0 & \text { otherwise }\end{cases}
$$


Obviously, $h_{n}(x) \in \mathcal{G}_{1}$ for each $n \geq 4$. With the aid of Lemma 4.4, we infer from (4-15) that

$$
\int_{R^{2}} \sum_{i, j=1}^{2} \frac{2 \varepsilon_{i j}\left(u_{0}\right) \varepsilon_{i j}\left(h_{n}\right)}{D\left(u_{0}\right)} d x \geq\left\langle G(x, 0), h_{n}(x)\right\rangle
$$

holds for every $n \geq 4$. It is easy to express $\varepsilon_{i j}\left(h_{n}\right)$ analogously to (4-8), (4-9) and (4-10) so that

$$
\sum_{i, j=1}^{2} \frac{2 \varepsilon_{i j}\left(u_{0}\right) \varepsilon_{i j}\left(h_{n}\right)}{D\left(u_{0}\right)}=\frac{(d f(r) / d r-f(r) / r)\left(d g_{n}(r) / d r-g_{n}(r) / r\right)}{|d f(r) / d r-f(r) / r|}
$$

which, together with (4-18), yields

$$
\begin{gathered}
2 \pi \int_{1}^{1+1 / n}\left(-n-\frac{n+1-n r}{r}\right) r d r+2 \pi \int_{1-1 / n}^{1}\left(-n+\frac{1-n+n r}{r}\right) r d r \\
\geq\left\langle G(x, 0), h_{n}(x)\right\rangle, \quad \text { for every } n \geq 4
\end{gathered}
$$

consequently,

$$
-4 \pi \geq\left\langle G(x, 0), h_{n}(x)\right\rangle \text { for every } n \geq 4 .
$$

In the meantime, $\left\langle G(x, 0), h_{n}(x)\right\rangle \rightarrow 0$ as $n \rightarrow \infty$, since $\left\|h_{n}(x)\right\|_{L^{2}\left(R^{2}\right)} \rightarrow 0$ as $n \rightarrow \infty$. This is a contraction, which proves the theorem.

5. Final remark. It is easy to show that Lemmas 1.1 through 1.7 and Lemma 2.4 are also valid in the three-dimensional case ((1-15) and (1-16) should be modified slightly). Renardy [9] has given a very elegant proof of Lemma 1.8 in any dimension. Therefore, Lemma 1.9 is still true for $R^{3}$. Renardy [9] has further shown that $\mathcal{G}_{1,0}=\mathcal{G}_{1}$ for $n=2,3$. Hence, we can obtain a similar result for the local existence and uniqueness of strong solutions in $R^{3}$ (according to our definition).

\section{REFERENCES}

1. G. Duvaut and J. L. Lions, Inequalities in mechanics and physics, Springer-Verlag, Berlin and New York, 1976.

2. C. Foias, C. Guillope and R. Temam, New a priori estimates for Navier-Stokes equations in dimension 3, Comm. Partial Differential Equations 6 (1981), 329-359.

3. A. Friedman, Partial differential equations, Krieger, New York, 1976.

4. T. Kato, Quasi-linear equations of evolution, with applications for partial differential equations, Lecture Notes in Math., vol. 448, Springer-Verlag, Berlin and New York, 1975, pp. 25-70.

5. J. L. Lions, Quelques méthodes de resolution des problèmes aux limites non linéaires, Dunod, Gauthier-Villars, Paris, 1969.

6. L. Schwartz, Theorie des distributions, Hermann, Paris, 1957.

7. R. Temam, Navier-Stokes equations, North-Holland, Amsterdam, 1979.

8. __ Navier-Stokes equations and nonlinear functional analysis, SIAM, Philadelphia, Pa., 1983.

9. M. Renardy, Dense imbedding of test functions in certain function spaces, Trans. Amer. Math. Soc. 298 (1986), 241-243.

Department of Mathematics, Virginia Polytechnic institute and State UNIVERSITY, BLACKSBURG, VIRGINIA 24060 